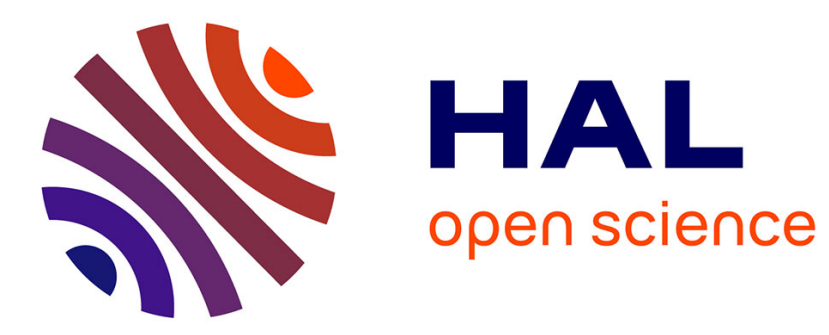

\title{
Nonsmooth modal analysis of a $N$-degree-of-freedom system undergoing a purely elastic impact law
}

\author{
Mathias Legrand, Stéphane Junca, Sokly Heng
}

\section{To cite this version:}

Mathias Legrand, Stéphane Junca, Sokly Heng. Nonsmooth modal analysis of a $N$-degree-of-freedom system undergoing a purely elastic impact law. Communications in Nonlinear Science and Numerical Simulation, 2017, 45, pp.190-219. 10.1016/j.cnsns.2016.08.022 . hal-01185980v2

\section{HAL Id: hal-01185980 \\ https://hal.science/hal-01185980v2}

Submitted on 7 Feb 2017

HAL is a multi-disciplinary open access archive for the deposit and dissemination of scientific research documents, whether they are published or not. The documents may come from teaching and research institutions in France or abroad, or from public or private research centers.
L'archive ouverte pluridisciplinaire HAL, est destinée au dépôt et à la diffusion de documents scientifiques de niveau recherche, publiés ou non, émanant des établissements d'enseignement et de recherche français ou étrangers, des laboratoires publics ou privés. 


\title{
Nonsmooth modal analysis of a $N$-degree-of-freedom system undergoing a purely elastic impact law
}

\author{
Mathias Legrand ${ }^{\dagger}$, Stéphane Junca ${ }^{\ddagger}$, and Sokly Heng ${ }^{\ddagger}$
}

\begin{abstract}
The dynamics of a $N$-degree-of-freedom autonomous oscillator undergoing an energy-preserving impact law on one of its masses is investigated in the light of nonlinear modal analysis. The impacted rigid foundation provides a natural Poincaré section of the investigated system from which is formulated a smooth First Return Map well-defined away from the grazing trajectory. In order to focus on the impact-induced nonlinearity, the oscillator is assumed linear. Continuous one-parameter families of $T$-periodic orbits featuring one impact per period and lying on two-dimensional invariant manifolds in the state-space are shown to exist. The geometry of these piecewise-smooth manifolds is such that a linear "flat" portion (on which contact is not activated) is continuously attached to a purely nonlinear portion (on which contact is activated once per period) exhibiting a velocity discontinuity through a grazing orbit. These features explain the newly introduced terminology "Nonsmooth modal analysis". The stability of the periodic orbits lying on the invariant manifolds is also explored by calculating the eigenvalues of the linearized First Return Map. Internal resonances and multiple impacts per period are not addressed in this work. However, the pre-stressed case is succinctly described and extensions to multiple oscillators as well as self-contact are discussed.
\end{abstract}

Key words nonlinear modal analysis; vibro-impact systems; frequency-energy plots; spectral stability analysis

1 Introduction $\quad 1$

2 Assumptions and formulations 3

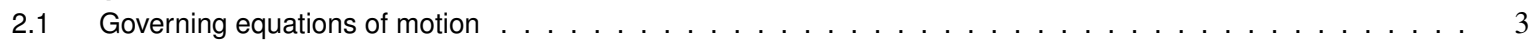

2.2 Periodic solutions with a single impact per period $\ldots \ldots \ldots \ldots \ldots \ldots \ldots \ldots$

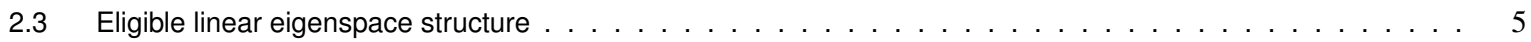

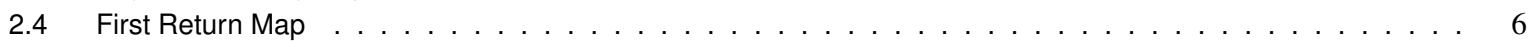

3 Main results $\quad 8$

4 Explicit solutions to the extended formulation $\quad 11$

5 Nonsmooth modes of vibration with one impact per period 13

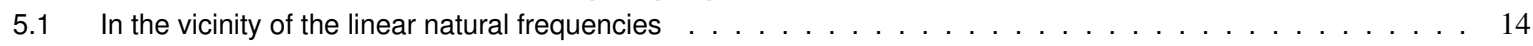

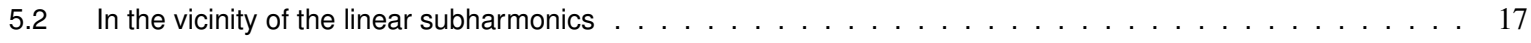

5.3 High Frequencies . . . . . . . . . . . . . . . . . . . . . . . . . . . . . . . . 19

6 Spectral stability analysis $\quad 20$

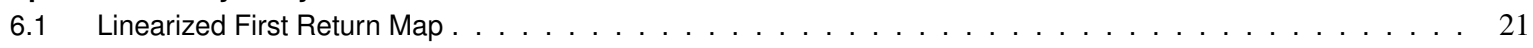

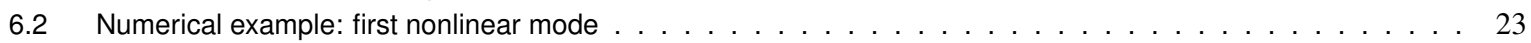

7 Pre-stressed configurations $\quad 23$

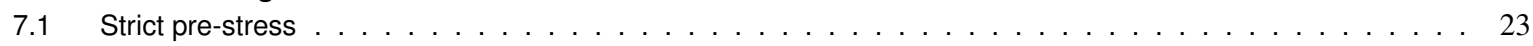

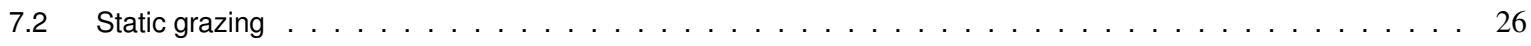

8 Multiple oscillators and self-contact $\quad 27$

9 Conclusion $\quad 28$

\begin{tabular}{lr} 
References & 30 \\
\hline
\end{tabular}

1. Introduction In the framework of linear vibration theory of conservative autonomous systems, natural modes of vibration, uniquely defined as a countable sequence of pairs of natural frequencies and corresponding normalized modeshapes, exhibit interrelated properties appealing to the engineer and mathematician: (1) they span the state-space through the principle of superposition, (2) they are invariant (that is linearly independent), orthogonal, and uncouple the equations of motion thus enabling the construction of reduced-order models, (3) they efficiently predict potential vibratory resonances of periodically forced systems [17].

Nonlinear modes of vibration (NLM) are conceived as conceptual extensions of linear modes when a nonlinear term arises in the governing equations. Their existence in the vicinity of fixed points is ensured by the center-stable manifold theorem [26]. For autonomous conservative non-resonant systems, they are defined as one-parameter continuous families of periodic orbits realized on two-dimensional curved manifolds in the state-space reducing to the common "flat" eigenspaces in the linear framework. Similarly

\footnotetext{
$\dagger$ McGill University, Department of Mechanical Engineering

$\ddagger$ Laboratoire J.A. Dieudonné, U.M.R. 6621 du CNRS, Université de Nice Sophia Antipolis

$\S$ Inria, Sophia Antipolis Méditerranée Research Centre, Project COFFEE
} 
to the invariance property of linear modes, such manifolds are also invariant under the flow: trajectories stemming from an initial condition in the manifold will remain in the manifold as time unfolds [26, 14]. On such manifolds, the modal dynamics can be regarded as a single degree-of-freedom second-order nonlinear oscillator from which all position and velocity coordinates can be functionally parametrized. For certain classes of systems, equivalence between nonlinear modal analysis and normal form theory is also established [29]. Evidently, the principle of superposition does no longer apply for nonlinear systems but NLM are useful for the analysis of mode bifurcations and forced resonances as well as for the construction of reduced-order models [31]. Such nonlinear oscillations are also known to exhibit energy-dependent natural frequencies and shapes [20], feature that is conveniently illustrated in frequency-energy plots (FEP) where they form the backbone of the nonlinear forced response curves [30].

The present work explores the free dynamics of $N$-degree-of-freedom (dof) vibro-impact oscillators within the nonlinear modal analysis framework. Such systems commonly consist of $N$ linearly coupled one-degree-of-freedom oscillators one of which undergoes a motion limiting constraint induced by the presence of a rigid foundation against which it impacts. The impenetrability condition is incorporated in the formulation through (1) an energy-preserving impact law acting on the impacting mass velocity and characterized by the restitution coefficient $e=1$ which introduces impulsive contact forces into the dynamics [5] and (2) a one-sided admissibility condition to be satisfied by the solution which has to be realized on the "appropriate" side of the rigid foundation. Between successive impacts, the motion is governed by a system of linear second-order ordinary differential equation in time with constant coefficients but the two previous conditions introduce nonlinearity into the dynamics. The above assumptions (one impact per period with $e=1$ ) might seem limiting but are motivated by the following considerations yielding mathematical simplicity:

1. Structural damping as well as well as a restitution coefficient $0 \leq e<1$ would destroy the targeted periodic orbits. Nonlinear modes of vibration involving damped oscillations are known to exist for smooth nonlinear systems but are more challenging to track [16].

2. As it is known in linear and smooth nonlinear modal analysis, modes of vibration of conservative autonomous systems accurately approximate the vibratory resonances of their periodically forced and slightly damped counterparts. This feature of interest to the designer is assumed to persist in the nonsmooth framework of this work.

3. As explained and demonstrated in the remainder, the domain of existence of the one-impact-per-period trajectories is far from being negligible.

It should be noted that the literature on (mainly low-dimensional) vibro-impact oscillators is vast (among many other references, see [9, 7, 1] for an overview). Nevertheless, it seems much more limited when it comes to the modal analysis of such systems. Three distinct formulations are then available: piecewise linear formulation [11, 12, 6], regularized unilateral formulation [19, 15], and purely nonsmooth formulation [23, 22]. Nonsmooth terms used to be replaced by simplified smooth approximations whose mathematical properties are better understood. This approach is acceptable in many situations but it is no longer justified to neglect such nonsmooth effects. In the, by now traditional, research area on nonsmooth systems, focus has been directed towards differential inclusions, convex analysis, or complementarity formulations that deal with standard questions like existence and uniqueness of solutions in general without special attention paid to continuous families of periodic solutions.

The paper is organized as follows: as explained in section 2, the proposed procedure involves the construction of a First Return Map where the switching hyperplane defined by the boundary of the rigid foundation impacted by the oscillator serves as a Poincare section in the state-space. Calculating the targeted families of periodic orbits reduces to determining the fixed points of this map. The corresponding main results are given in section 3. In section 4, (quasi-) closed-form solutions are provided but the first return time has to be approximated numerically. Admissible trajectories defining nonlinear modes of vibration are discussed in section 5. Stability together with an introductory bifurcation analysis are then undertaken by computing the eigenvalues of the linearized First Return Map numerically evaluated at a fixed-point in section 6 . The pre-stressed case (that is with a negative algebraic clearance) involving new vibratory features is considered in section 7. Self-contact and multi-oscillators are quickly tackled through a simple change of variables in section 8 . 


\section{Assumptions and formulations}

2.1. Governing equations of motion A generic, initially unstressed, autonomous and conservative $N$-degree-of-freedom oscillator, similar in essence to the one illustrated in Fig. 2.1, is investigated. The $N$

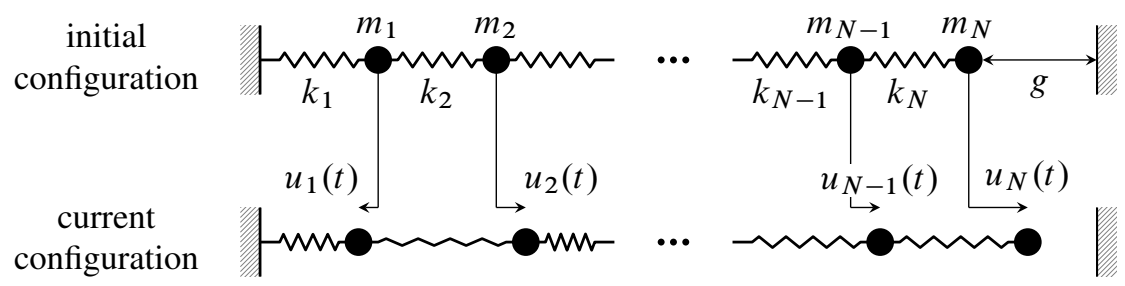

Figure 2.1: Investigated $N$-degree-of-freedom vibratory system subject to a unilateral contact constraint on $u_{N}(t)$

masses $m_{i}$ and stiffnesses $k_{i}, i=1, \ldots, N$ are associated to the $N$ degrees-of-freedom, or displacements, $u_{i}(t)$ where $t$ stands for time. The last degree-of-freedom $u_{N}(t)$ is subject to an impenetrability condition due to the presence of a rigid foundation located at a distance $g$ from its equilibrium ${ }^{1}$. The corresponding equations governing the dynamics of the system shall be expressed in the compact matrix form

$$
\mathbf{M u}+\mathbf{K u}+\mathbf{f}^{\mathrm{c}}=\mathbf{0}
$$

where $\mathbf{M} \in \mathbb{R}^{N, N}$ and $\mathbf{K} \in \mathbb{R}^{N, N}$ are the mass and stiffness matrices of the system, respectively. Displacements are stored in vector $\mathbf{u}=\left[u_{1}, \ldots, u_{N-1}, u_{N}\right]^{\top} \in \mathbb{R}^{N}$ and vector $\mathbf{f}^{\mathrm{c}}=\left[0, \ldots, 0, f_{N}^{\mathrm{c}}\right]^{\top} \in$ $\mathbb{R}^{N}$ stands for the contact force emerging from the unilateral condition. At this point, the initial conditions in displacement $\mathbf{u}$ and velocity $\dot{\mathbf{u}}$ are not discussed.

Unilateral contact constraints are commonly expressed as complementarity conditions such that the contact force vector $\mathbf{f}^{\mathrm{c}}$ in Eq. (2.1) becomes

$$
\begin{aligned}
& \mathbf{M u ̈}+\mathbf{K u}-\lambda \mathbf{B}^{\top}=\mathbf{0} \\
& \mathbf{B u}-g \leq 0 ; \lambda \leq 0 \quad ; \quad \lambda(\mathbf{B u}-g)=0
\end{aligned}
$$

where matrix $\mathbf{B} \in \mathbb{R}^{1, N}$ maps the displacement vector $\mathbf{u}$ to the $N$ th displacement $u_{N}$, that is $\mathbf{B}=\mathbf{e}_{N}^{\top}=$ $\left(\begin{array}{llll}0 & 0 & \ldots & 0\end{array}\right)$. It is well-known that formulation $(2.2)$ is ill-posed $[8,5,4]$ and the complementarity conditions should be supplemented with an impact law governing the dynamics during impact. In this work, a simple energy-conserving Newton's impact law in the form

$$
u_{N}(t)=g \quad \Rightarrow \quad \dot{u}_{N}^{+}(t)=-\dot{u}_{N}^{-}(t)
$$

is implemented with the following notations

$$
\dot{u}_{N}^{+}(t) \stackrel{\text { def }}{=} \lim _{0<\epsilon \rightarrow 0} \dot{u}_{N}(t+\epsilon) \quad \text { and } \quad \dot{u}_{N}^{-}(t) \stackrel{\text { def }}{=} \lim _{0<\epsilon \rightarrow 0} \dot{u}_{N}(t-\epsilon)
$$

in mind. It is understood that $\dot{u}_{N}$, the velocity of the $N$ th degree-of-freedom, is a function of bounded variations. Usually, the restitution coefficient $e$ belongs to the interval $[0 ; 1]$ with the impact rule $\dot{u}_{N}^{+}(t)=-e \dot{u}_{N}^{-}(t)$. Since we are targeting periodic motions without impact dissipation, $e=1$ in (2.3) by assumption.

Following $[1,2,3]$, the formulation of the problem of interest now takes the form: Find the displacement $\mathbf{u}(t)$ and the contact force $\lambda(t)$ satisfying

$$
\begin{aligned}
& \mathbf{M u ̈}+\mathbf{K u}=\lambda \mathbf{B}^{\top}, \\
& \lambda \text { is a non-positive measure, } \\
& u_{N}(t)-g \leq 0, \quad \forall t, \\
& \text { suppr } \lambda \subset\left\{t ; u_{N}(t)=g\right\}, \\
& u_{N}(t)=g \Rightarrow \dot{u}_{N}^{+}(t)=-\dot{u}_{N}^{-}(t) .
\end{aligned}
$$

The measure $\lambda$ depends on the solution $\mathbf{u}$. It arises as a nonlinear impulsive restoring force in (2.5a).

\footnotetext{
$1 \mathrm{~g}$ is the algebraic distance between the equilibrium position of mass $N$ and the rigid wall. It is negative with pre-stress.
} 
2.2. Periodic solutions with a single impact per period This work targets one-parameter continuous families of periodic solutions. If such families exist, it is expected that they will be organized on two-dimensional invariant manifolds which usually characterize modes of vibration for nonlinear mechanical systems [14, 11, 23].

A major assumption is made: trajectories experience a single impact per period where the period $T$ is to be found. Since the system of interest is autonomous, the phase is free and formulation (2.5) can be advantageously simplified by considering, without loss of generality, that the impact occurs at $t=T$.

Remark 1. As already said, it might look highly limiting to assume one impact per period on a single degree-of-freedom. However, in continuum mechanics, it should be noted that the contact interface is only a small portion of the boundary of the system. Also, as we will see in the remainder, the one-impactper-period solutions are organized on many "non-local" invariant manifolds in the phase-space. As a consequence, a broad range of potential applications in rigid-body dynamics is expected. For instance, applications in robotics shall be foreseen: machining robots are known to be flexible and might, under specific operating conditions, start to interact with the processed mechanical system, through vibro-impact mechanisms. Also, an assembly task performed with a robot might involve intermittent contact and it becomes crucial to simulate the task accurately for vibratory prediction purposes [24].

Accordingly, the problem shall be reformulated and simplified as follows (see [6] for a similar formulation for piecewise linear systems):

Definition 2.1 [Extended formulation] Find $T$ and $\mathbf{u}(t)$ such that:

$$
\begin{aligned}
& \mathbf{M u ̈}+\mathbf{K u}=\mathbf{0}, \quad \forall t \in] 0 ; T[ \\
& \mathbf{u}(T)=\mathbf{u}(0) \\
& u_{N}(0)=g \\
& \dot{\mathbf{u}}^{-}(T)=\mathbf{S}^{+}(0)
\end{aligned}
$$

where $\mathbf{S}$ is the matrix of symmetry with respect to the hyperplane $\dot{u}_{N}=0$, or jump matrix:

$$
\mathbf{S}=\left[\begin{array}{cccc}
1 & 0 & \cdots & 0 \\
0 & \ddots & \ddots & \vdots \\
\vdots & \ddots & 1 & 0 \\
0 & \cdots & 0 & -1
\end{array}\right]
$$

System (2.6) says the following:

- Away from an impact, the system is free and its dynamics governed by Eq. (2.6a).

- The displacement is a continuous and periodic function of time.

- The period $T$ is an unknown of the problem.

- At the beginning $t=0$ of the period, the impacting mass is located on the rigid wall.

- As reflected by the matrix $\mathbf{S}$, the velocities of masses 1 to $N-1$ are continuous functions of time while the velocity of mass $N$ is discontinuous at $t=T$ where it satisfies $\dot{u}_{N}^{+}(T)=-\dot{u}_{N}^{-}(T)$. They are all periodic functions of time.

In (2.6), the measure $\lambda$ does not seem to arise explicitly. In fact, it emerges as the zero measure in (2.6a) when $u_{N}(t)-g<0$ and as a Dirac measure at $t=T$ such that $u_{N}(T)=g$ in (2.6d) which defines its magnitude. Also, the less restrictive formulation (2.6) exhibits more $T$-periodic solutions than (2.5) because the unilateral condition $u_{N}(t)-g<0$ might be violated on interval ]0; $T$ [: their admissibility will have to be systematically scrutinized. Still, this extended formulation can be completely solved through closed-form expressions as detailed later.

Definition 2.2 [Admissible solution] A solution to the extended formulation (Definition 2.1) is said to be admissible if

$$
\forall t, \quad u_{N}(t) \leq g
$$


2.3. Eligible linear eigenspace structure The construction of the nonlinear periodic solutions is tightly related to the eigenspace structure of the underlying linear mechanical system for which the unilateral contact constraint is discarded. In Eq. (2.1), matrices $\mathbf{M}$ and $\mathbf{K}$ are assumed to be positive definite thus excluding rigid-body motions. Accordingly, there exists a matrix $\mathbf{P}$ of $\mathbf{M}$-orthogonal eigenmodes which diagonalizes both the mass and stiffness matrices, that is

$$
\begin{aligned}
& \mathbf{P}^{\top} \mathbf{M P}=\mathbf{I}_{N} \\
& \mathbf{P}^{\top} \mathbf{K P}=\boldsymbol{\Omega}^{2}=\left.\operatorname{diag}\left(\omega_{i}^{2}\right)\right|_{i=1, \ldots, N}
\end{aligned}
$$

where $\mathbf{I}_{N}$ is the identity matrix in $\mathbb{R}^{N, N}$ and $\omega_{i}^{2}, i=1, \ldots, N$ are the $N$ eigenfrequencies of the underlying linear system. In the remainder, it is assumed that eigenfrequencies are all distinct, $i e$

$$
0<\omega_{1}<\ldots<\omega_{N} \quad \text { and } \quad 0<T_{N}<\ldots<T_{1}
$$

where $\omega_{i} T_{i}=2 \pi, i=1, \ldots, N$. In order to efficiently expose the coming developments, a definition based on the modal matrix $\mathbf{P}$ is needed:

Definition 2.3 [Interaction coefficient] For all $j=1, \ldots, N$, the quantity:

$$
a_{j}=P_{N j} P_{j N}^{-1}
$$

is called the interaction coefficient between the displacement of mass $N$ along the $j$ th linear mode and the unilateral contact constraint.

It should be noted that the interaction coefficients satisfy

$$
\sum_{j=1}^{N} a_{j}=\mathbf{e}_{N}^{\top} \mathbf{P} \mathbf{P}^{-1} \mathbf{e}_{N}=1 .
$$

Assumption 2.1 None of the $N$ interaction coefficients shall vanish, that is $a_{j} \neq 0, j=1, \ldots, N$.

Mechanically, this simply means that the last mass always experiences a displacement along a linear modal motion. Further interpretations will be provided in the remainder of the paper.

Definition 2.4 [Linear grazing orbit] A linear grazing orbit associated to the $j$ th linear mode is a periodic trajectory $\mathbf{u}$ such that

$$
\max _{t \in \mathbb{R}} u_{N}(t)=g .
$$

Assumption 2.1 implies that $P_{N j} \neq 0, j=1, \ldots, N$ which also reflects the existence and uniqueness of the $j$ th linear grazing orbit

$$
\mathbf{u}_{j}(t)=\frac{g}{P_{N j}} \cos \left(\omega_{j} t\right) \mathbf{P}_{\cdot j}
$$

where $\mathbf{P}_{\cdot j}$ is column $j$ of matrix $\mathbf{P}$. In other words, the last row $\mathbf{e}_{N}^{\top} \mathbf{P}=\mathbf{P}_{N}$. of the eigenvectors matrix has no vanishing terms. Hence, a linear grazing orbit is automatically admissible since $u_{N}(t) \leq g$ for all time $t$. Since $\mathbf{P}^{-1}=\mathbf{P}^{\top} \mathbf{M}$, Assumption 2.1 also entails

$$
P_{j N}^{-1}=\mathbf{e}_{j}^{\top} \mathbf{P}^{-1} \mathbf{e}_{N}=\mathbf{e}_{j}^{\top} \mathbf{P}^{\top} \mathbf{M} \mathbf{e}_{N}=\left(\mathbf{M} \mathbf{e}_{N}\right)^{\top} \mathbf{P} \mathbf{e}_{j} \neq 0, \quad j=1, \ldots, N
$$

where $\mathbf{e}_{j}^{\top}=(0, \ldots, 0,1,0, \ldots, 0) \in \mathbb{R}^{N}$ is a row vector with a 1 located at its $j$ th coordinate. In particular, if the mass matrix is the constant diagonal matrix $\mathbf{M}=m \mathbf{I}_{N}$ with $m>0$ then $\mathbf{M e}_{N}=m \mathbf{e}_{N}$, $P_{j N}^{-1}=m P_{N j}$, and $a_{j} \neq 0$ reduces to $P_{N j} \neq 0$. For a diagonal mass matrix, the sign of the interaction coefficients is known through Assumption 2.1

$$
\mathbf{M}=m \mathbf{I}_{N} \quad \Rightarrow \quad a_{j}=m P_{N j}^{2}>0, \quad j=1, \ldots, N .
$$


Remark 2. In this work, all numerical examples and corresponding illustrations are provided for the generic oscillator depicted in Fig. 2.1 that is

$$
\mathbf{M}=\left[\begin{array}{ccccc}
m & 0 & 0 & 0 & 0 \\
0 & m & 0 & 0 & 0 \\
0 & 0 & m & 0 & 0 \\
0 & 0 & 0 & m & 0 \\
0 & 0 & 0 & 0 & m
\end{array}\right] \quad \text { and } \quad \mathbf{K}=\left[\begin{array}{ccccc}
2 k & -k & 0 & 0 & 0 \\
-k & 2 k & -k & 0 & 0 \\
0 & -k & 2 k & -k & 0 \\
0 & 0 & -k & 2 k & -k \\
0 & 0 & 0 & -k & k
\end{array}\right] \text {. }
$$

with $N=5, m_{j}=m=1 / N$, and $k_{j}=k=N, i=j, \ldots, N$. As such, it falls into the framework explained above. Yet, the proposed construction of nonsmooth modes of vibration is still valid for a much broader class of discrete oscillators.

2.4. First Return Map The admissible state-space of the investigated system takes the form

$$
\mathcal{D}=\mathcal{D}^{\circ} \cup H^{+} \cup H^{-} \cup H^{0}
$$

where

$$
\begin{aligned}
\mathcal{D}^{\circ} & =\left\{(\mathbf{u}, \dot{\mathbf{u}}) \in \mathbb{R}^{2 N} \text { such that } u_{N}<g\right\}, \\
H^{+} & =\left\{\left(\mathbf{u}, \dot{\mathbf{u}}^{+}\right) \in \mathbb{R}^{2 N} \text { such that } u_{N}=g \text { and } \dot{u}_{N}^{+}<0\right\}, \\
H^{-} & =\left\{\left(\mathbf{u}, \dot{\mathbf{u}}^{-}\right) \in \mathbb{R}^{2 N} \text { such that } u_{N}=g \text { and } \dot{u}_{N}^{-}>0\right\}, \\
H^{0} & =\left\{(\mathbf{u}, \dot{\mathbf{u}}) \in \mathbb{R}^{2 N} \text { such that } u_{N}=g \text { and } \dot{u}_{N}=0\right\} .
\end{aligned}
$$

It is illustrated in Fig. 2.2.

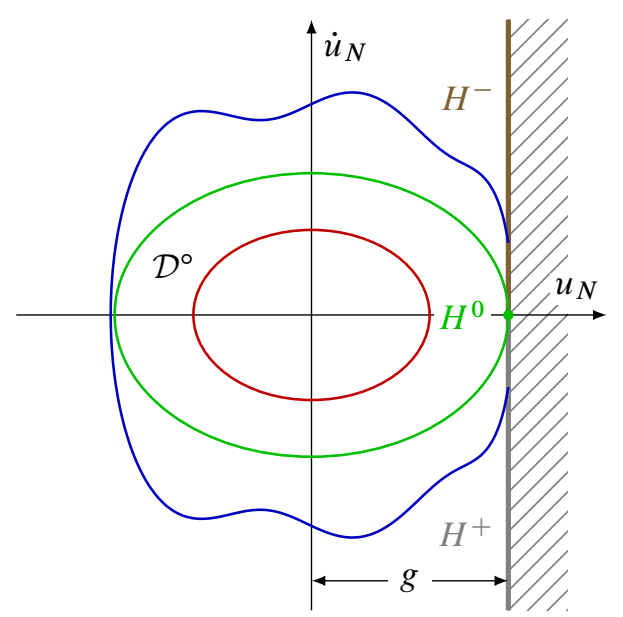

Figure 2.2: Cross-section of the admissible state-space. The state-space is partitioned into two regions separated by the hypersurface $H=H^{+} \cup H^{0} \cup H^{-}$. The hatched area is the zone of non-admissible displacements, and its complementary $\mathcal{D}^{\circ}$ in the state-space is populated by admissible orbits of which they are three types: one admissible periodic orbit with one impact per period [blue], one admissible periodic orbit with no impact (linear system) [red], and one grazing periodic orbit [green]

Seeking solutions of the extended formulation 2.1 can be equivalently seen as finding fixed-points of a First Return Map, the so-called Poincaré map. Among all eligible Poincaré cross-sections, the half hyperplane $H^{+}$plays a crucial role in the impact dynamics and is selected. Loosely speaking, it is expected that the Poincare map is well defined from $H^{+}$to $H^{+}$. Indeed, we prove later that near an admissible periodic solution with initial data in $\mathrm{H}^{+}$, the First Return Map is locally well-defined and smooth. Since our focus is on periodic solutions with a unique impact per period, trajectories $(\mathbf{u}, \dot{\mathbf{u}})$ defined as follows

$$
H^{+} \rightarrow \mathcal{D}^{\circ} \rightarrow H^{-} \rightarrow H^{+}
$$

are considered. More challenging yet not addressed situations shall emerge ${ }^{2}$ with potential grazing impacts on $H^{0}$ between two impacts on $H^{+}$as illustrated in Fig. 5.2.

\footnotetext{
2 Trajectories with more than one impact per period are investigated in [28] for a two-degree-of-freedom system.
} 
In order to enjoy an almost closed-form expression of the Poincaré map, Eq. (2.6a) is projected onto the linear modal space through the transformation $\mathbf{u}=\mathbf{P q}$ to become

$$
\mathbf{I}_{N} \ddot{\mathbf{q}}+\mathbf{\Omega}^{2} \mathbf{q}=\mathbf{0}
$$

by invoking Eq. (2.9). Assume the existence of the first return time $T$ from $H^{+}$to $H^{-}$which is a nonlinear function of the initial state $\left(\mathbf{u}(0), \dot{\mathbf{u}}^{+}(0)\right)$ of the system. It is then straightforward to map the state of system at $t=0^{+}$to its state at $t=T^{-}$by solving the linear differential system $(2.21)[6,22]$ as follows:

$$
\begin{aligned}
& \mathbf{q}^{-}(T)=\cos (T \Omega) \mathbf{q}^{+}(0)+\boldsymbol{\Omega}^{-1} \sin (T \Omega) \dot{\mathbf{q}}^{+}(0) \\
& \dot{\mathbf{q}}^{-}(T)=-\boldsymbol{\Omega} \sin (T \boldsymbol{\Omega}) \mathbf{q}^{+}(0)+\cos (T \Omega) \dot{\mathbf{q}}^{+}(0)
\end{aligned}
$$

where $\cos (T \boldsymbol{\Omega}) \equiv \boldsymbol{d i a g}\left(\cos \left(\omega_{i} T\right)_{i=1, \ldots, N}\right)$ and $\sin (T \boldsymbol{\Omega}) \equiv \boldsymbol{d i a g}\left(\sin \left(\omega_{i} T\right)_{i=1, \ldots, N}\right)$ are used as notations. In modal coordinates, the continuity of the displacement as well as the jump in velocity occurring at $t=T$ read

$$
\begin{aligned}
\mathbf{q}^{+}(T) & =\mathbf{q}^{-}(T) \\
\dot{\mathbf{q}}^{+}(T) & =\mathcal{S} \dot{\mathbf{q}}^{-}(T)
\end{aligned}
$$

where the expression of the jump matrix in modal coordinates is

$$
\mathcal{S}=\mathbf{P}^{-1} \mathbf{S P} \text {. }
$$

When $T$ is known and by inserting Eq. (2.22) into Eq. (2.23), a $2 N \times 2 N$ Return Map matrix $\mathbf{R}(T)$ mapping the system from $t=0^{+}$to $t=T^{+}$can be built in modal coordinates. It includes the free flight in $\mathcal{D}^{\circ}$ as well as one impact on $H^{-}$.

Definition 2.5 [First Return Map] The modal state of the system at time $t$ is denoted by the quantity $\mathbf{Q}(t)=\left(\mathbf{q}(t), \dot{\mathbf{q}}^{+}(t)\right)=\left(\mathbf{P}^{-1} \mathbf{u}(t), \mathbf{P}^{-1} \dot{\mathbf{u}}^{+}(t)\right)$. If $\left(\mathbf{u}(0), \dot{\mathbf{u}}^{+}(0)\right) \in H^{+}, T=T(\mathbf{Q}(0))>0$ is the first return time to $H^{-}$where $\mathbf{Q}(0)$ is the initial modal state of the system. Then, the modal state at time $T^{+}$is given by the First Return Map

$$
\mathbf{F}(\mathbf{Q}(0))=\mathbf{Q}(T)=\mathbf{R}(T(\mathbf{Q}(0))) \mathbf{Q}(0)
$$

with

$$
\mathbf{R}(T)=\left[\begin{array}{cc}
\cos (T \Omega) & \Omega^{-1} \sin (T \Omega) \\
-\mathcal{S} \Omega \sin (T \Omega) & \mathcal{S} \cos (T \Omega)
\end{array}\right]
$$

To better "visualize" the expression of the First Return Map, Eq. (2.25) is expanded as

$$
\left(\begin{array}{c}
\mathbf{q}(T) \\
\dot{\mathbf{q}}^{+}(T)
\end{array}\right)=\left[\begin{array}{cc}
\cos (T \Omega) & \boldsymbol{\Omega}^{-1} \sin (T \Omega) \\
-\mathcal{S} \Omega \sin (T \Omega) & \mathcal{S} \cos (T \Omega)
\end{array}\right]\left(\begin{array}{c}
\mathbf{q}(0) \\
\dot{\mathbf{q}}^{+}(0)
\end{array}\right)
$$

From the periodicity conditions (2.6b) and (2.6d), the extended formulation (Definition 2.1) is transformed into a nonlinear fixed point problem: find $\mathbf{Q}(0)$ such that $\mathbf{F}(\mathbf{Q}(0))=\mathbf{Q}(0)$ also arising in the form of Eq. (2.25) as

$$
\mathbf{Q}(0)=\mathbf{R}(T(\mathbf{Q}(0))) \mathbf{Q}(0) \quad \Leftrightarrow \quad\left(\mathbf{R}(T(\mathbf{Q}(0)))-\mathbf{I}_{2 N}\right) \mathbf{Q}(0)=\mathbf{0}_{2 N} .
$$

Determining the fixed-points of the First Return Map thus reduces to solving a generalized eigenvalue problem in $(T, \mathbf{Q}(0))$. Since $\mathbf{Q}(0) \in H^{+}$, which is a half hyperplane of dimension $2 N-1$, there are $2 N=1+2 N-1$ unknowns for $2 N$ equations. Although the scalar $T$ is an implicit function of the $2 N-1$ dimensional variable $\mathbf{Q}(0)$, the key point to solve nonlinear system (2.28) is to fix $T$ such that system (2.28) is then linear in $\mathbf{Q}(0)$. The constraint $\mathbf{Q}(0) \in H^{+}$yields a unique solution (the proof is 
given later). In other words, we obtain a one-to-one relation between the period and the periodic solution with one impact per period ${ }^{3}$.

We might think that the First Return Map is a linear map, but this is a wrong statement. First, the first return time $T$ from $H^{+}$to $H^{-}$is an unknown of the nonlinear transcendental algebraic equation $u_{N}(T)=$ $g$. Second, since $\mathcal{S}$ is a non diagonal matrix, all the linear modes are fully coupled through (2.23b), which is a well known feature of nonlinearity.

Let us emphasize the expression of the impact nonlinearity in terms of the matrix $\mathcal{S}$ and the interaction coefficients $a_{j}$. It is clear that $\mathbf{S}$ is a diagonal matrix but $\mathcal{S}$ is not. In the unrealistic case where $\mathcal{S}=\mathbf{I}_{N}$ (which is strictly impossible), the reflection on $\mathrm{H}^{-}$is removed and the problem would be simply linear. In other words, the nonlinearity shall be quantified by the difference $\mathcal{S}-\mathbf{I}_{N}$ which is directly linked to the interaction coefficients $a_{j}$ from definition 2.3. The identity $\mathbf{S}=\mathbf{I}_{N}-2 \mathbf{e}_{N} \mathbf{e}_{N}^{\top}$ yields

$$
\mathcal{S}=\mathbf{I}_{N}-2 \mathbf{P}^{-1} \mathbf{e}_{N} \mathbf{e}_{N}^{\top} \mathbf{P}=\mathbf{I}_{N}-2 \mathbf{P}_{. N}^{-1} \mathbf{P}_{N}^{\top}
$$

The matrix $\mathbf{e}_{N} \mathbf{e}_{N}^{\top}$ is the rank one matrix of the orthogonal projection onto $\mathbf{e}_{N}$. The interaction coefficients are the diagonal terms of the rank one matrix $\mathbf{P}_{\cdot N}^{-1} \mathbf{P}_{N}^{\top}$. uniquely defined by the last row of the eigenvector matrix and the last column of its inverse

$$
a_{j}=\left(\mathbf{P}^{-1} \mathbf{e}_{N} \mathbf{e}_{N}^{\top} \mathbf{P}\right)_{j j}=\left(\mathbf{P}_{\cdot N}^{-1} \mathbf{P}_{N}^{\top} \cdot\right)_{j j} .
$$

The condition $a_{j} \neq 0$ from assumption 2.1 simply means that the diagonal of the projector on $\mathbf{e}_{N}$ expressed in the modal basis $\mathbf{P}_{. N}^{-1} \mathbf{P}_{N}^{\top}$. does not vanish. Accordingly, all the coefficients of this matrix are nonzero since it is a rank one matrix. More precisely, the condition $a_{j}=0$ has a mechanical interpretation:

- if $P_{j N}^{-1}=0$, the entire row $j$ of $\left(\mathbf{P}_{. N}^{-1} \mathbf{P}_{N}^{\top}\right.$.) vanishes. Thus, equation $j$ of $(2.23 \mathrm{~b})$ becomes $q_{j}^{+}(T)=$ $q_{j}^{-}(T)$ which means that the linear mode $j$ is not coupled to any of the other modes $k \neq j$ on $H^{-}$.

- if $P_{N j}=0$, none of the modes $k \neq j$ are affected by mode $j$ on $H^{-}$since the $N$ th mass does not move along linear mode $j$.

3. Main results This paper is mainly concerned with the construction of nonlinear modes of vibration of a conservative and autonomous $N$-degree-of-freedom impact oscillator. Such modes are defined as continuous families of periodic solutions with one impact per period. These periodic solutions are found to be organized on two-dimensional invariant manifolds in the state-space. The proposed formulation gives access to (almost) closed-form solutions, admissibility criteria, as well as spectral stability (and bifurcation) of these nonlinear modes.

Remark 3. In this contribution, derivations are mostly expressed in terms of the period $T$ but the plots are displayed in terms of $\omega=2 \pi / T$ in line with conventional Frequency-Energy Plots. As stated in [14], "[a] nonlinear modal motion is represented by a point in Frequency-Energy Plots, which is drawn at a frequency corresponding to the minimal period of the periodic motion and at an energy equal to the conserved total energy during the motion. A branch is a family of modal motions possessing the same qualitative features."

A majority of the proposed results are related to the key function

$$
w_{N}(T)=\sum_{j=1}^{N} a_{j} \phi_{j}(T), \quad \phi_{j}(T)=\frac{\sin \left(\omega_{j} T\right)}{\omega_{j}\left(1-\cos \left(\omega_{j} T\right)\right)}=\frac{1}{\omega_{j} \tan \left(\omega_{j} T / 2\right)}
$$

illustrated in Fig. 3.1. The existence and uniqueness of solutions to the extended formulation (Definition 2.1) is proven as soon as $0<\left|w_{N}(T)\right|<+\infty$.

Remark 4. The function $w_{N}$ is a barycenter of the functions $\phi_{j}$ through (2.12).

Remark 5. The poles of $w_{N}$, for which $\left|w_{N}(T)\right|=\infty$, are all the linear periods and their subharmonics, that is $T \in \cup_{j=1}^{N} T_{j} \mathbb{N}$ (see Fig. 3.1).

\footnotetext{
3 The solution to Eq. (2.28) is depicted in the form of a Frequency-Energy plot in Fig. 3.2: it is the union of the thin grey and thick black lines. As long as the non-admissible solutions are not discarded, the system is shown to exhibit a continuous spectrum.
} 


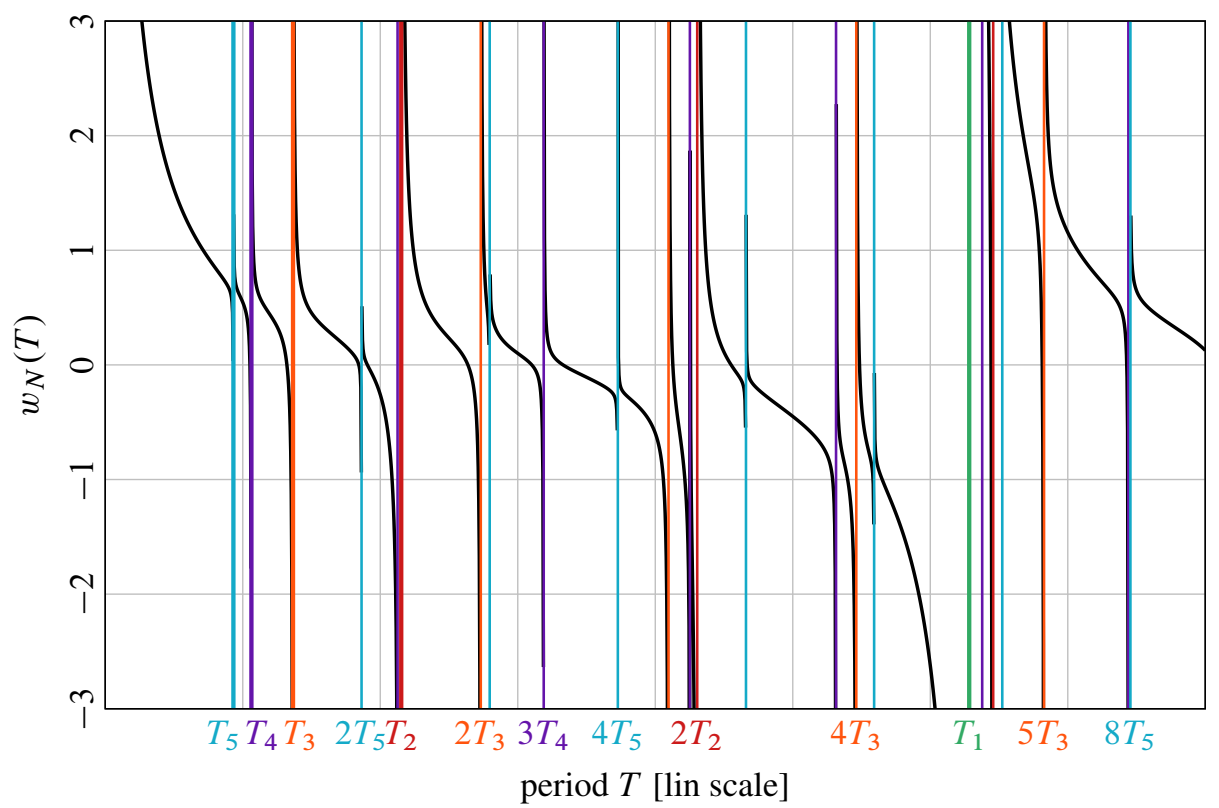

Figure 3.1: Function $w_{N}$ versus period $T$ for the system of interest. The linear periods and corresponding subharmonics $k T_{i}, i=1, \ldots, 5, k \in \mathbb{N}^{*+}$ [coloured vertical lines] are the poles of this function. All $T$ such that $w_{N}(T)=0$ are the zeros of the function

Remark 6. The function $w_{N}$ admits at most a countable set of zeros.

Assumption 3.1 [No internal resonance assumption] The linear periods of the system of interest satisfy $\bigcap_{j=1}^{N} T_{j} \mathbb{N}=\emptyset$.

Through assumption 3.1, difficulties induced by internal resonances are discarded. We then have the following results:

Theorem 3.1 [Extended formulation: existence and uniqueness of solutions] Assume a positive $T$ that is neither a pole nor a zero of $w_{N}$, then for all such $T$ :

- The extended formulation (Definition 2.1) admits one and only one solution $t \mapsto \mathbf{u}(t ; T)^{4}$.

- The set of initial data

$$
\mathcal{V}_{0}=\left\{\left(\mathbf{u}(0 ; T), \dot{\mathbf{u}}^{+}(0 ; T)\right), 0<T, 0<\left|w_{N}(T)\right|<+\infty\right\}
$$

for the extended formulation is an analytic curve which generates a set of periodic solutions forming a piecewise analytic manifold of dimension 2 defined as

$$
\mathcal{V}=\left\{\left(\mathbf{u}(t ; T), \dot{\mathbf{u}}^{+}(t ; T)\right), 0 \leq t \leq T, 0<T, 0<\left|w_{N}(T)\right|<\infty\right\}
$$

in the state-space $\mathbb{R}^{2 N}$.

- If both assumptions 2.1 and 3.1 are satisfied, then the Lipschitz extension $\mathbf{u}(\cdot ; T)$ when $T \rightarrow T_{j}$ is the linear grazing solution denoted $\mathbf{u}\left(\cdot ; T_{j}\right)$ here.

Note that the analytic curve is not connected when $w_{N}(T)=0$. When assumption 3.1 holds and $w_{N}(T)= \pm \infty$, then there is an analytic connection with the initial data associated to a linear grazing orbit.

Solutions to the extended formulation are not necessarily admissible solutions since many of them violate the non-penetration condition (2.8): they are called "phantom solutions". A one-sided condition naturally arises near the linear period $T_{j}[23]$.

Theorem 3.2 [One-sided condition for admissible periodic solutions] Assumptions 2.1 and 3.1 hold. Then, for all $T>0$ such that $T \approx T_{j}$ and $a_{j}\left(T-T_{j}\right)<0$ :

- The unique T-periodic solution $\mathbf{u}(\cdot ; T)$ of Theorem 3.1 is admissible.

\footnotetext{
${ }^{4}$ When there is no ambiguity, i.e. for a fixed $T$, the quantity $\mathbf{u}(t ; T)$ will be simply denoted $\mathbf{u}(t)$.
} 
- The invariant manifold of admissible periodic solutions with one impact per period near the linear grazing orbit is a piecewise smooth manifold in the admissible state-space $\mathcal{D}^{\circ}$.

Moreover:

- For $a_{j}\left(T-T_{j}\right)>0$ and $T \approx T_{j}$, the solution of the extended formulation is not admissible.

- If for all $j, a_{j}>0$ then the admissible frequencies are bounded.

The extended formulation (Definition 2.1) also exhibits solutions near linear subharmonics. The question of their admissibility is more challenging since in the most general case, a numerical verification is required. Still, a simple criterion for subharmonics of order one-half exists.

Theorem 3.3 [Admissible solutions near linear subharmonics of order one-half] Assumptions 2.1 and 3.1 hold. Then, for $T \approx 2 T_{j}$ such that $a_{j}\left(T-2 T_{j}\right)<0$ and $\mathbf{u}(T / 2 ; T) \leq g$, the solution $t \mapsto \mathbf{u}(t ; T)$ is admissible.

For subharmonics of lower order $(1 / 3,1 / 4, \ldots)$, the admissibility is discussed in Section 5.2.

Theorems 3.1, 3.2 and 3.3 are illustrated in Fig. 3.2 for the system of interest. It should be noted that this system features only strictly positive interaction coefficients $a_{j}, j=1, \ldots, 5$, stemming from Eq. (2.16): from a mechanical standpoint, only stiffening is observed when contact is initiated. The solution branches of the extended formulation are separated by the zeros and poles of $w_{N}$. "Ghost" branches which correspond to inadmissible solutions have to be ignored. Note that their boundaries are always a grazing admissible solution or a solution with infinite energy (zero of $w_{N}$ ).

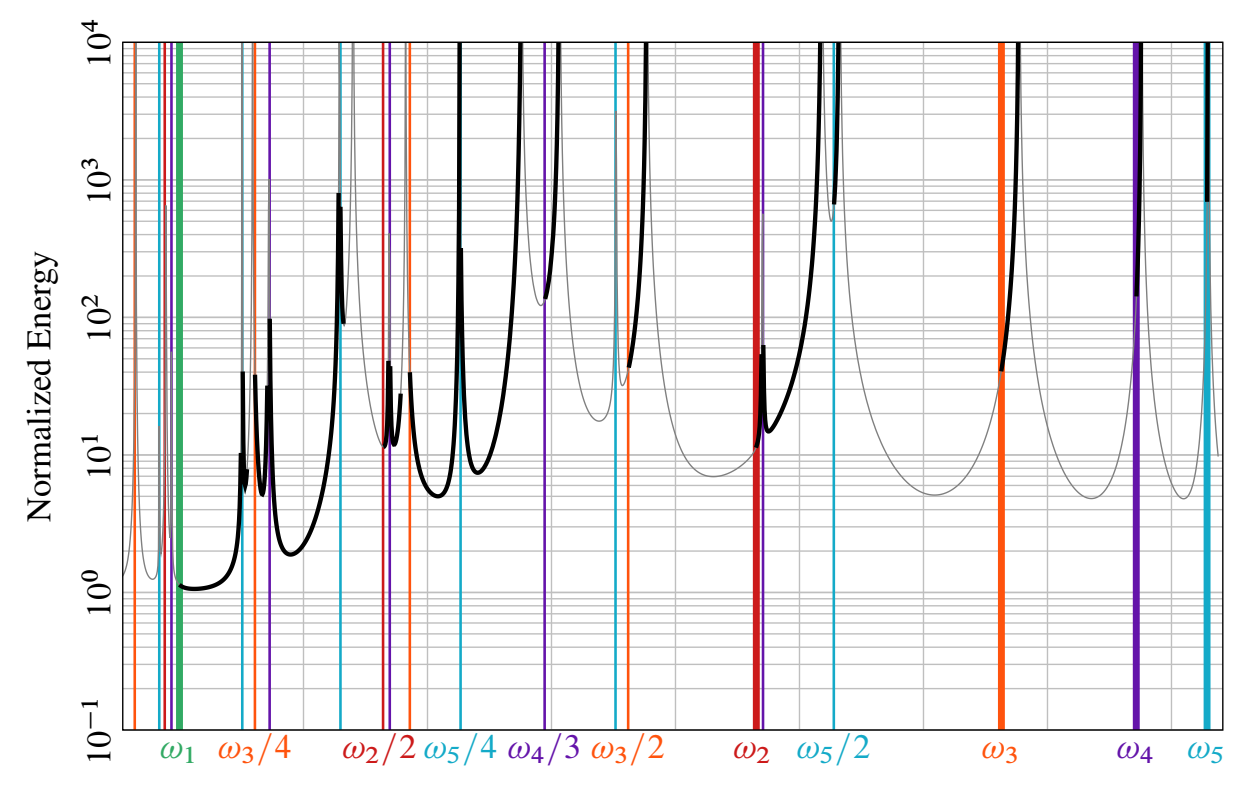

Frequency of vibration $\omega$ [log scale]

Figure 3.2: Frequency-Energy Plot: non-admissible spectrum [thin gray line] and admissible spectrum [thick black line]. Linear eigenfrequencies $\omega_{i}, i=1, \ldots, 5$ [thick vertical colored lines] as well as the corresponding subharmonics $\omega_{i} / k, i=1, \ldots, 5, k=1, \ldots, 10$ [thin vertical colored lines] are displayed. The frequency interval $\omega \in\left[0 ; \omega_{1}\right]$ features admissible branches that are not shown for the sake of clarity

The key-tool for the stability analysis is the First Return Map whose fixed-points can be expressed in a closed form as soon as the first return time $T$ is (numerically) known. Moreover, in the vicinity of an impacting periodic orbit, the First Return Map is locally well defined and a fully explicit differential of this map is also available. Stability can then be evaluated numerically by computing its eigenvalues. The corresponding results are expressed in modal coordinates for conciseness where $\mathbf{Q}(t ; T)=\left(\mathbf{q}(t ; T), \dot{\mathbf{q}}^{+}(t ; T)\right)$ denotes a $T$-periodic solution. We also choose the notations $\overline{\mathbf{e}}_{N}^{\top}=(0, \ldots, 0,1,0, \ldots, 0) \in \mathbb{R}^{2 N}\left(\overline{\mathbf{e}}_{N}^{\top}\right.$ is a row vector with a 1 located at the $N$ th coordinate $)$ and $\overline{\mathbf{P}}=\mathbf{B d i a g}(\mathbf{P}, \mathbf{P})$. For grazing impact, it is already known that the First Return Map is not differentiable and the grazing solutions are not expected to be stable [21].

Corollary 3.1 [First Return Map near an impacting periodic orbit] Let $T_{0} \notin \bigcup_{j=1}^{N} T_{j} \mathbb{N}$ be a nonlinear period and $\mathbf{Q}_{0}=\mathbf{Q}\left(0 ; T_{0}\right) \in H^{+}$be the initial condition generating an orbit of period $T_{0}=T\left(\mathbf{Q}_{0}\right)$. 
Then, for all $\mathbf{Q} \simeq \mathbf{Q}_{0} \in H^{+}$, the first return time $T(\mathbf{Q})$ and the First Return Map $\mathbf{F}(\mathbf{Q})$ are well defined and smooth. Moreover, the gradient of the first return time and the matrix of the linearized First Return Map at $\mathbf{Q}_{0}$ are respectively given by

$$
\begin{aligned}
\nabla T\left(\mathbf{Q}_{0}\right) & =\frac{w_{N}\left(T_{0}\right)}{g} \overline{\mathbf{e}}_{N}^{\top} \overline{\mathbf{P}} \mathbf{R}\left(T_{0}\right), \\
\mathbf{D F}\left(\mathbf{Q}_{0}\right) & =\mathbf{R}\left(T_{0}\right)+\dot{\mathbf{R}}\left(T_{0}\right)\left[\mathbf{Q}_{0} \nabla T\left(\mathbf{Q}_{0}\right)\right] .
\end{aligned}
$$

Furthermore, 1 is an eigenvalue of $\mathbf{D F}\left(\mathbf{Q}_{0}\right)$.

Numerical examples seem to show that 1 is a simple eigenvalue as opposed to usual (smooth) Hamiltonian systems for which 1 is a double eigenvalue [18]. Another derivation of $\mathbf{D F}$ in the space variables is proposed in Appendix A.

4. Explicit solutions to the extended formulation In this section, existence and uniqueness of solutions for the extended formulation (Definition 2.1) are proven. Closed-form initial data generating periodic solutions with one impact per period is derived for all nonlinear periods $T \notin \bigcup_{j=1}^{N} T_{j} \mathbb{N}$ and the associated invariant manifolds are investigated. Admissibility is discussed later.

The following quantities are well defined:

$$
\begin{aligned}
\boldsymbol{\Phi}(T) & =\boldsymbol{\Omega}^{-1}(\mathbf{I}-\cos (T \boldsymbol{\Omega}))^{-1} \sin (T \boldsymbol{\Omega}) \\
\mathbf{w}(T) & =\left(\mathbf{P} \boldsymbol{\Phi}(T) \mathbf{P}^{-1}\right) \mathbf{e}_{N} \\
w_{N}(T) & =\mathbf{e}_{N}^{\top} \mathbf{w}(T)
\end{aligned}
$$

Notice that $w_{N}$ is exactly the function defined in (3.1). A first important result is established:

\section{Proposition 4.1 [Zero velocities, analytic manifolds, and symmetry]}

1. For all $T \notin \bigcup_{j=1}^{N} T_{j} \mathbb{N}$, there exists a unique solution $t \mapsto \mathbf{u}(t ; T)$ to the extended formulation with period $T$ given by its initial data

$$
\begin{aligned}
\mathbf{u}(0 ; T) & =\dot{u}_{N}^{+}(0 ; T) \mathbf{w}(T) \\
\dot{\mathbf{u}}^{+}(0 ; T) & =\dot{u}_{N}^{+}(0 ; T) \mathbf{e}_{N}
\end{aligned}
$$

where $\dot{u}_{N}^{+}(0 ; T)=g / w_{N}(T)$.

2. The initial data set of solutions is an analytic manifold as soon as $0<\left|w_{N}(T)\right|<+\infty$ and the set of periodic solutions is a piecewise analytic invariant manifold in the state-space.

3. The solution is symmetric with respect to $T / 2$ that is $\mathbf{u}(T / 2+t ; T)=\mathbf{u}(T / 2-t ; T), \forall t$ and $\dot{\mathbf{u}}(T / 2 ; T)=0$.

Before proving Proposition 4.1, we explicitly express the eigenspace associated to the eigenvalue 1 of $\mathbf{R}(T)$ from Eq. (2.26).

Lemma 4.1 [Fixed-points of the Linear Return Map R] If $T \notin \bigcup_{j=1}^{N} T_{j} \mathbb{N}$ then system (2.28) defines a one-dimensional vector space parametrized by $c \in \mathbb{R}$ :

$$
\left(\begin{array}{c}
\mathbf{u}(0) \\
\dot{\mathbf{u}}^{+}(0)
\end{array}\right)=\left[\begin{array}{cc}
\mathbf{P} & \mathbf{0} \\
\mathbf{0} & \mathbf{P}
\end{array}\right]\left(\begin{array}{c}
\mathbf{q}(0) \\
\dot{\mathbf{q}}^{+}(0)
\end{array}\right)=c\left(\begin{array}{c}
\mathbf{w}(T) \\
\mathbf{e}_{N}
\end{array}\right)
$$

Note that all velocities except velocity $N$ are zero when the $N$ th mass hits the wall, as already known for a two degree-of-freedom system [22]. The solution in Eq. (4.3) can be seen as the "shape" $\mathbf{w}(T)$ of the mode (as commonly found for linear modes that are standing waves) to which is added an initial velocity on the $N$ th degree-of-freedom.

Proof. The kernel $\operatorname{ker}\left(\mathbf{R}(T)-\mathbf{I}_{2 N}\right)$ can be explicitly expressed through a block-manipulation of $\mathbf{R}(T)$ detailed below:

$$
\begin{aligned}
& {\left[\begin{array}{cc}
\cos (T \Omega)-\mathbf{I}_{N} & \boldsymbol{\Omega}^{-1} \sin (T \Omega) \\
-\mathcal{S} \boldsymbol{s} \sin (T \Omega) & \mathcal{S} \cos (T \Omega)-\mathbf{I}_{N}
\end{array}\right] \sim\left[\begin{array}{cc}
\mathbf{I}_{N}-\cos (T \Omega) & -\boldsymbol{\Omega}^{-1} \sin (T \Omega) \\
-\boldsymbol{\Omega} \sin (T \Omega) & \cos (T \Omega)-\mathcal{S}
\end{array}\right] } \\
\sim & {\left[\begin{array}{cc}
\mathbf{I}_{N}-\cos (T \Omega) & -\boldsymbol{\Omega}^{-1} \sin (T \Omega) \\
\mathbf{0} & \left(\mathbf{I}_{N}-\cos (T \Omega)\right)(\cos (T \Omega)-\mathcal{S})-\sin ^{2}(T \Omega)
\end{array}\right] }
\end{aligned}
$$


where $\sim$ stands for "equivalent homogeneous systems". The lower-right block can be simplified as follows:

$$
\begin{aligned}
\left(\mathbf{I}_{N}-\right. & \cos (T \Omega))(\cos (T \Omega)-\mathcal{S})-\sin ^{2}(T \Omega) \\
& =-\left(\mathbf{I}_{N}-\cos (T \Omega)\right) \mathcal{S}+\cos (T \Omega)-\cos ^{2}(T \Omega)-\sin ^{2}(T \Omega) \\
& =-\left(\mathbf{I}_{N}-\cos (T \Omega)\right) \mathcal{S}+\left(\cos (T \Omega)-\mathbf{I}_{N}\right) \\
& =-\left(\mathbf{I}_{N}-\cos (T \Omega)\right)\left(\mathcal{S}+\mathbf{I}_{N}\right) \\
& =-\left(\mathbf{I}_{N}-\cos (T \Omega)\right) \mathbf{P}^{-1}\left(\mathbf{S}+\mathbf{I}_{N}\right) \mathbf{P} .
\end{aligned}
$$

By assumption, the matrix $\mathbf{I}_{N}-\cos (T \Omega)$ is invertible and system (4.4) is equivalent to

$$
\left[\begin{array}{cc}
\mathbf{I}_{N}-\cos (T \Omega) & -\mathbf{\Omega}^{-1} \sin (T \Omega) \\
\mathbf{0} & \left(\mathbf{S}+\mathbf{I}_{N}\right) \mathbf{P}
\end{array}\right]
$$

Since $\dot{\mathbf{u}}=\mathbf{P} \dot{\mathbf{q}}$, the right lower block in (4.6) yields $\left(\mathbf{S}+\mathbf{I}_{N}\right) \dot{\mathbf{u}}=\mathbf{0}$, that is $\dot{\mathbf{u}}=c \mathbf{e}_{N}$ with $c \in \mathbb{R}$. Similarly, the upper block yields $\mathbf{q}=c \boldsymbol{\Phi}(T) \mathbf{P}^{-1} \mathbf{e}_{N}$.

We now prove Proposition 4.1 and Theorem 3.1.

Proof. The identity $c=\dot{u}_{N}^{+}(0)$ is a direct consequence of the last equality of (4.3) which means that the outcoming velocity at the boundary parametrizes every periodic solution. If $w_{N}(T) \neq 0$, condition (2.6c) implies

$$
\dot{u}_{N}^{+}(0)=\frac{g}{w_{N}(T)} .
$$

Due to the velocity jump, only piecewise regularity of $\mathcal{V}$ can be expected. Nevertheless, the projection of $\mathcal{V}$ on the displacement

$$
\mathcal{V}_{\mathbf{u}}=\left\{\mathbf{u}(t ; T), 0 \leq t \leq T, 0<T, 0<\left|w_{N}(T)\right|<\infty\right\}
$$

is at least continuous since the free flight response is governed by a linear differential system and is accordingly very smooth. The regularity of the manifold $\mathcal{V}$ can be completely identified through the restriction of $\mathcal{V}$ to the Poincaré section

$$
\mathcal{V}_{0}=\left\{\left(\mathbf{u}(0 ; T), \dot{\mathbf{u}}^{+}(0 ; T)\right), 0<T, 0<\left|w_{N}(T)\right|<\infty\right\} .
$$

Singularities of $\mathcal{V}_{0}$ shall arise when $T$ approaches the poles $\bigcup_{j=1}^{N} T_{j} \mathbb{N}$ of $w_{N}$. Consider the case $T \rightarrow T_{j}$. The only singular term in $\mathbf{w}(T)$ stems from the matrix $\left(\mathbf{I}_{N}-\cos (T \Omega)\right)^{-1}$ through assumption 3.1. More precisely, only the diagonal term

$$
\phi_{j}(T)=\frac{\sin \left(\omega_{j} T\right)}{\omega_{j}\left(1-\cos \left(\omega_{j} T\right)\right)} \sim \frac{2}{\omega_{j}^{2}\left(T-T_{j}\right)}
$$

becomes singular when $T$ approaches $T_{j}$ since all linear periods are distinct. From assumption $2.1, w_{N}(T)$ is precisely expanded as

$$
w_{N}(T)=\sum_{k=1}^{N} a_{k} \phi_{k}(T) \sim a_{j} \phi_{j}(T) \quad \text { when } \quad T \rightarrow T_{j}
$$

and the condition $u_{N}(0 ; T)=g=\dot{u}_{N}^{+}(0 ; T) w_{N}(T)$ yields

$$
\dot{u}_{N}^{+}(0 ; T) \sim \frac{g}{a_{j} \phi_{j}(T)} \sim \frac{g}{2} \frac{\omega_{j}^{2}}{a_{j}}\left(T-T_{j}\right) .
$$

Thus $\dot{u}_{N}^{+}(0 ; T) \rightarrow 0$ when $T \rightarrow T_{j}$ : the manifold $\mathcal{V}_{0}$ is smooth at $T=T_{j}$ and recovers the initial data of the corresponding linear grazing orbit. Let $\mathbf{e}_{k}$ be the vector in $\mathbb{R}^{N}$ such that all coordinates are 0 except the $k$ th one equal to 1 . The computation is straightforward:

- if $P_{k j} \neq 0$ :

$$
w_{k}(T)=\mathbf{e}_{k}^{\top} \mathbf{w}(T)=\sum_{p=1}^{N} P_{k p} P_{p N}^{-1} \phi_{p}(T) \sim P_{k j} P_{j N}^{-1} \phi_{j}(T) \quad \text { when } \quad T \rightarrow T_{j}
$$

and thus

$$
u_{k}(0 ; T)=\dot{u}_{N}^{+}(0 ; T) w_{k}(T)=g w_{k}(T) / w_{N}(T) \rightarrow g P_{k j} / P_{N j}, \quad k=1, \ldots, N
$$

and $\dot{\mathbf{u}}^{+}(0 ; T) \rightarrow \mathbf{0}$ which forms the initial data of the linear grazing mode. 
- if $P_{k j}=0$, then $w_{k}(T)=\mathcal{O}(1)$ so $u_{k}(0 ; T) \rightarrow 0$ and the previous statement still holds true. Moreover, the function $T \mapsto\left(T-T_{j}\right) \phi_{j}(T)$ is analytic at $T=T_{j}$ and then $\mathcal{V}_{0}$ is an analytic manifold.

The same investigation is valid for the subharmonics $T \simeq k T_{j}, k \in \mathbb{Z}^{*}$.

Finally we prove the symmetry of the solutions. The proof only uses the uniqueness initial data theorem for linear differential system (without contact). To this end, let us define $\mathbf{z}(t):=\mathbf{u}(T / 2+t),-T / 2<t<T / 2$ : it is a solution of the linear differential system and can be extended to all time $t \in \mathbb{R}$. This vectorial function is very smooth and also well defined at $t= \pm T / 2$ with $\mathbf{z}(T / 2)=\mathbf{z}(-T / 2)=\mathbf{u}(0)$ and $\dot{\mathbf{z}}(T / 2)=-\dot{\mathbf{z}}(-T / 2)=$ $-\dot{\mathbf{u}}^{+}(0)$. As a consequence, $\mathbf{z}(t)$ and $\mathbf{z}(-t)$ satisfy the same linear differential system with the same initial data, that is $\mathbf{z}(t)=\mathbf{z}(-t), \forall t \in \mathbb{R}$. As a direct consequence, $\dot{\mathbf{z}}(0)=0$ that is $\mathbf{u}(t)=\mathbf{u}(-t), \forall|t| \leq T / 2$ and $\dot{\mathbf{u}}(T / 2)=\mathbf{0}$, which concludes the proof.

5. Nonsmooth modes of vibration with one impact per period The previous section defines the set of all solutions to the extended formulation (Definition 2.1). They are not all physically acceptable and the admissible solutions only form the so-called "Nonsmooth modes of vibration".

Among all the admissible branches shown in Fig. 3.2, special attention is paid to four of them illustrated in Fig. 5.1 since they embed the main properties of all branches: they are defined in the vicinity of the fundamental and sub-harmonics of the linear modes and their domain of definition is either bounded by a nonlinear grazing orbit (branches 1,2, and 3) or not bounded (branch 4). These nonlinear grazing

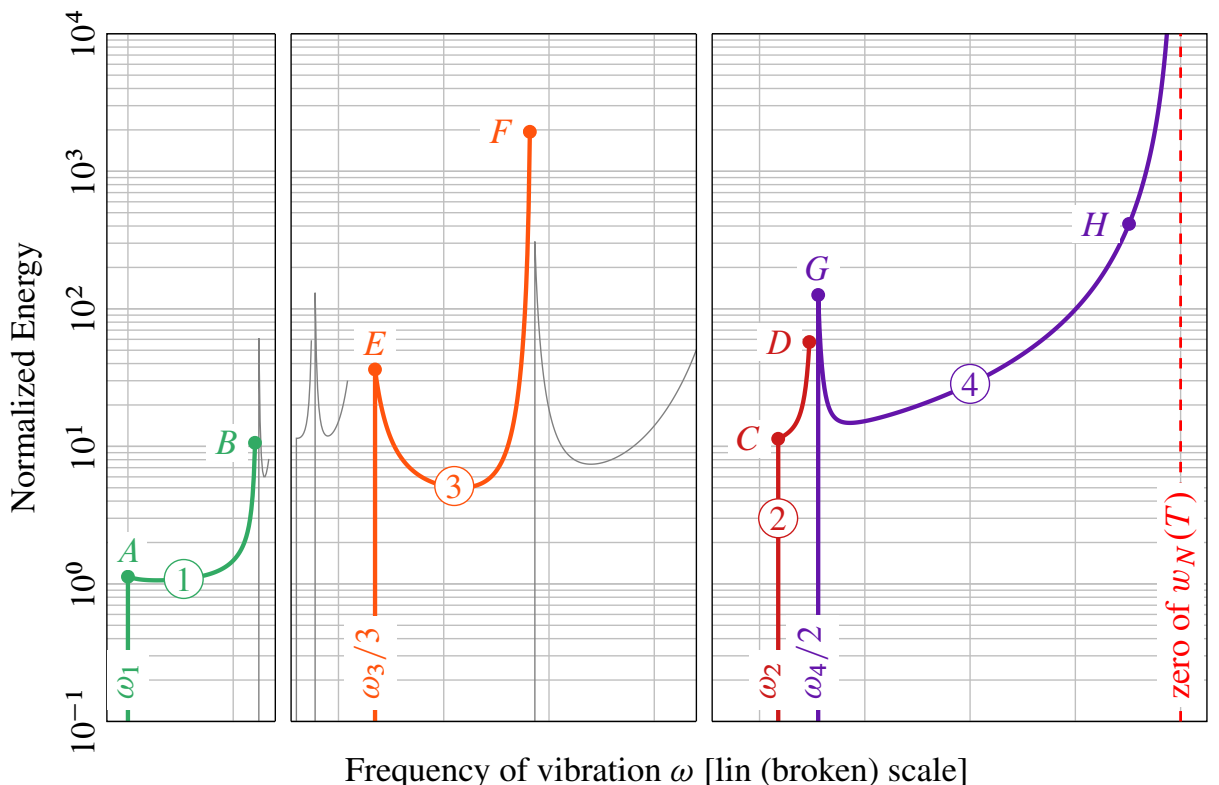

Figure 5.1: Zoomed-in Frequency-Energy plot: investigated branches of the admissible spectrum [see Fig. 3.2]. Colored vertical straight segments are the linear portions of the nonlinear modes of vibration [contact not activated] while the colored curved segments are the nonlinear portions [one impact per period] of the nonlinear modes of vibration: the corresponding supporting manifolds are depicted in figures 5.4, 5.8, 5.5, and 5.9 with increasing frequencies. Points $A, C, E$, and $G$ correspond to the linear grazing orbits in these figures, while points $B, D$, and $F$ correspond to the nonlinear grazing orbits. One zero of $w_{N}(T)$ is highlighted

orbits are plotted in Fig. 5.2, together with their linear counterparts. It should be understood that these are solutions where the system (probably, as not further explored in this work) bifurcates from one impact per period orbits to multiple impacts per period orbits. The proposed approach is not capable of investigating these new types of response and a sophisticated formulation tackling more than one impact per period is needed [28].

All illustrated invariant manifolds are projected onto the coordinates $\left(u_{N-1}, u_{N}, \dot{u}_{N}\right)$ of the statespace as generically shown in Fig. 5.3. The terminology "Hyperplane view" and "Configuration view" used later is self-explanatory. The jump in velocity induced by the impact law is highlighted by a thick black line representing the one-dimensional manifold $\mathcal{V}_{0}$. 


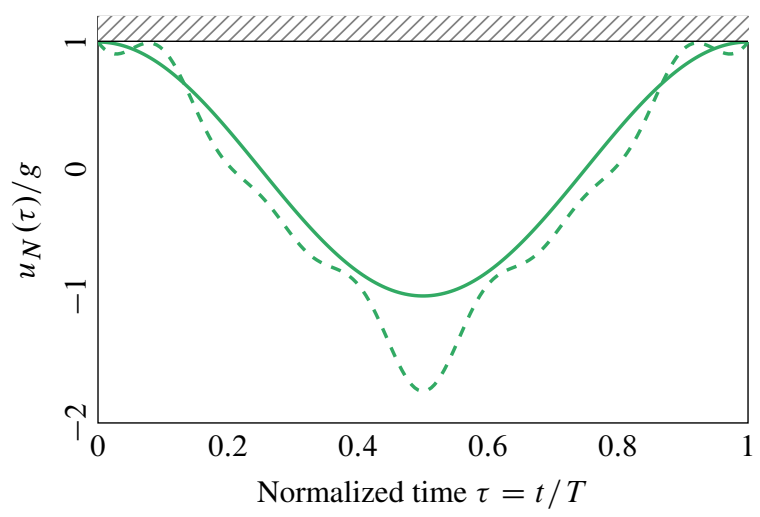

(a) Branch 1: points $A$ and $B$

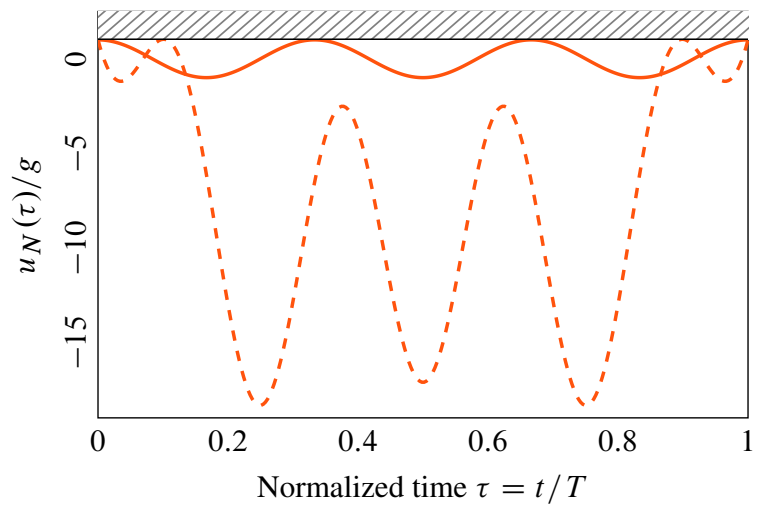

(c) Branch 3: points $E$ and $F$

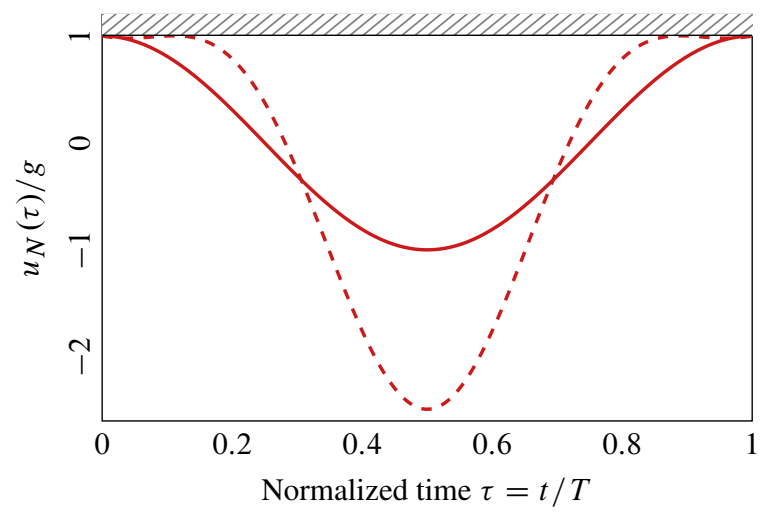

(b) Branch 2: points $C$ and $D$

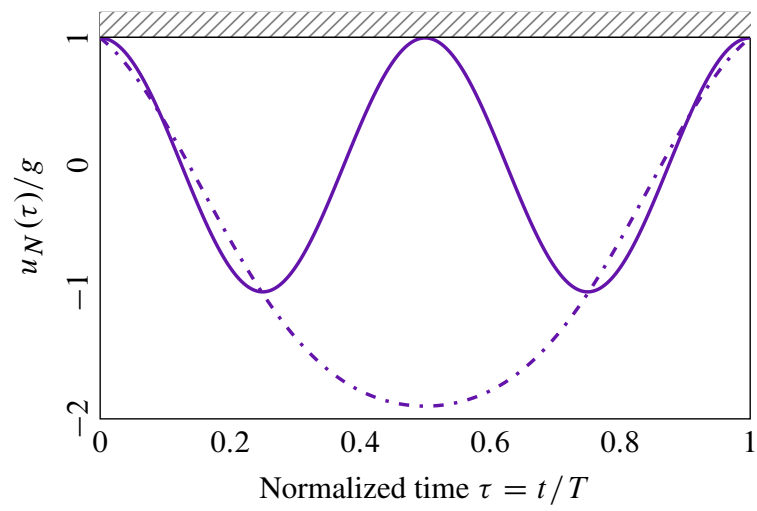

(d) Branch 4: points $G$ and $H$ (not a grazing orbit)

Figure 5.2: Linear [solid line] and nonlinear [dashed line] grazing displacements mentioned in Fig. 5.1. Nonlinear grazing orbits feature additional grazing impact instants thus limiting the domain of admissibility of one impact per period orbits. Note that the time axis is rescaled for each orbit for viewing purposes

5.1. In the vicinity of the linear natural frequencies The solution of the eigenvalue problem (2.28) given in Proposition 4.1 has various consequences on the existence and admissibility of a periodic solution satisfying $u_{N}(0)=g$ and $\dot{u}_{N}^{+}(0)<0$.

Proposition 5.1 [Admissibility] Assumptions 2.3, 3.1, and $T \notin \bigcup_{j=1}^{N} T_{j} \mathbb{N}$ hold. Then a periodic solution $\mathbf{u}(\cdot ; T)$ will be an admissible solution if:

1. Necessary admissibility conditions: $\dot{u}_{N}^{+}(0)<0$ and $w_{N}(T)<0$.

2. Sufficient admissibility condition: $a_{j}\left(T-T_{j}\right)<0$ and $T \simeq T_{j}$.

Remark 7. The existence of a branch of admissible periodic solutions near a linear grazing mode pertains to grazing bifurcation mechanisms near periodic orbits [21,7].

This proposition is a reformulation of Theorem 3.2 which is proven below.

Proof. The first necessary condition $\dot{u}_{N}^{+}(0 ; T) \leq 0$ should be satisfied otherwise mass $N$ penetrates the rigid wall. Since $\dot{u}_{N}^{+}(0 ; T) \neq 0$ for the nonlinear period $T$, the condition $\dot{u}_{N}^{+}(0 ; T) \leq 0$ becomes $\dot{u}_{N}^{+}(0 ; T)<0$. The second necessary condition stems from the fact that $\mathbf{u}(0 ; T)=c \mathbf{w}(T)$ with $c=\dot{u}_{N}^{+}(0 ; T)$ and $0<g=$ $u_{N}(0 ; T)=c w_{N}(T)$.

Equivalent (4.12) for $T \simeq T_{j}$ implies the one-sided condition $a_{j}\left(T-T_{j}\right)<0$. Assume a positive interaction coefficient $a_{j}$. The admissibility of a $T$-periodic solution $u_{N}(t ; T)$ with only one impact per period means

$$
\left.u_{N}(t ; T)<g, \quad \forall t \in\right] 0 ; T[.
$$

A continuity argument on $\mathbf{u}(t ; T)$ along $t$ and $T$ belonging to the compact set $K_{\epsilon}=\{(t, T), 0 \leq t \leq$ $\left.T, T_{j}-\epsilon \leq T \leq T_{j}\right\}$ parametrized by $0<\epsilon \ll 1$ shows that $\mathbf{u}(t ; T) \approx \mathbf{u}\left(t ; T_{j}\right)$ which is the linear grazing solution satisfying $u_{N}\left(t ; T_{j}\right)<g$ except for $t=0$ and $t=T$ : as a consequence, $u_{N}(t ; T)<g$ except for $t \approx 0$ and $t \approx T$. Let us investigate inequality (5.1) around $t \approx 0$ only since the other configuration 


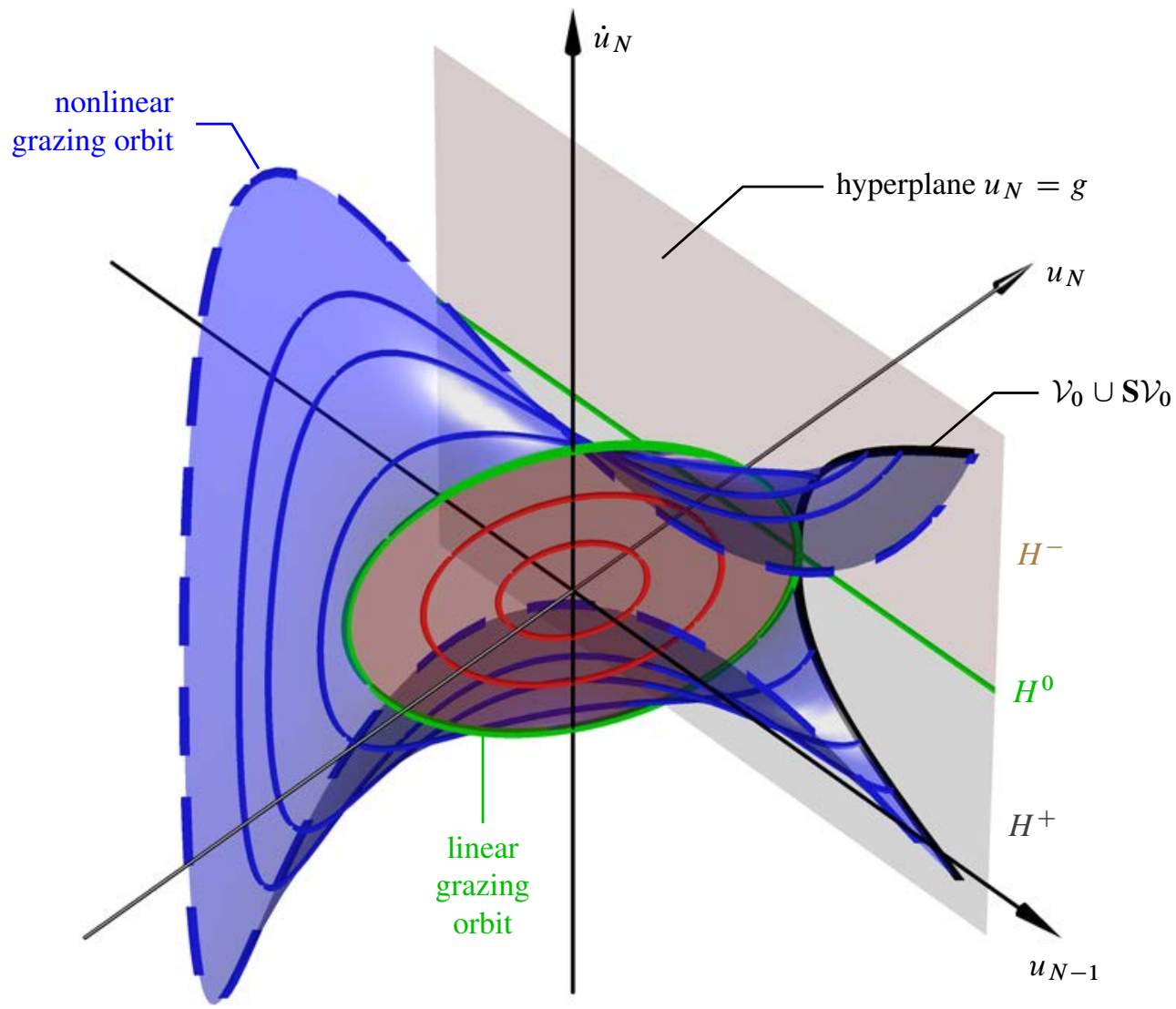

Figure 5.3: Generic admissible manifold for illustration purposes. It shows (1) the linear portion [red] for which contact is not activated during one period and (2) the nonlinear portion [blue] supporting periodic orbits with one impact per period. The linear grazing orbit joins the two portions defining the manifold. The continuum of periodic orbits "hits" the hyperplane $u_{N}=g$ along the one-dimensional manifold $\mathcal{V}_{0} \cup \mathbf{S} \mathcal{V}_{0}$ [thick black line]

is handled in an identical fashion. The solutions $u_{N}(0 ; T)=g$ for $T<T_{j}$ have negative velocity on the wall $\dot{u}_{N}^{+}(0 ; T)<0$ without any uniform estimate since $\dot{u}_{N}(0 ; T) \rightarrow 0$ when $T \rightarrow T_{j}$. We cannot conclude directly on the existence of a time zone ]0; $t_{0}$ [ independent of $T$ such that $u_{N}(t ; T)<g$ for all $(t, T) \in K_{\epsilon}$, $0<t<t_{0}$. Fortunately, there exist $t_{0}>0$ and $\epsilon>0$ such that

$$
\sup _{\left[0 ; t_{0}\right] \times\left[T_{j}-\epsilon ; T_{j}\right]} \ddot{u}_{N}(t ; T)<0
$$

which is a sufficient condition to ensure admissibility because $\dot{u}_{N}(t ; T)$ decreases on $\left[0 ; t_{0}\right]$ and $\dot{u}_{N}(0 ; T) \leq 0$ so $\dot{u}_{N}(t ; T)<0$ on $\left.] 0 ; t_{0}\right] \times\left[T_{j}-\epsilon ; T_{j}\right]$, and then $u_{N}(t ; T)<g$ on $\left.] 0 ; t_{0}\right] \times\left[T_{j}-\epsilon ; T_{j}\right]$. Let us calculate the second derivatives by starting with

$$
\mathbf{P}^{-1} \mathbf{P}=\mathbf{I} \quad \Leftrightarrow \quad \sum_{p=1}^{N} P_{k p}^{-1} P_{p j}=\delta_{k j} .
$$

The expansions are now performed in a neighborhood of $T_{j}$ through (4.10), (4.11), and (4.14) when $T \rightarrow T_{j}$. For $0<t<T$, the explicit solution is

$$
\mathbf{u}(t)=\mathbf{P} \cos (t \boldsymbol{\Omega}) \mathbf{P}^{-1} \mathbf{u}(0)+\mathbf{P} \boldsymbol{\Omega}^{-1} \sin (t \boldsymbol{\Omega}) \mathbf{P}^{-1} \dot{\mathbf{u}}^{+}(0)
$$

which leads to the expressions for

- the displacement:

$$
\begin{aligned}
& u_{N}(t ; T)=\frac{g}{w_{N}(T)}\left(\sum_{\ell=1}^{N} \sum_{k=1}^{N} P_{N \ell} P_{\ell k}^{-1} \cos \left(\omega_{\ell} t\right) w_{k}(T)+\sum_{k=1}^{N} a_{k} \frac{\sin \left(\omega_{k} t\right)}{\omega_{k}}\right) \\
& u_{N}(0 ; T)=g,
\end{aligned}
$$




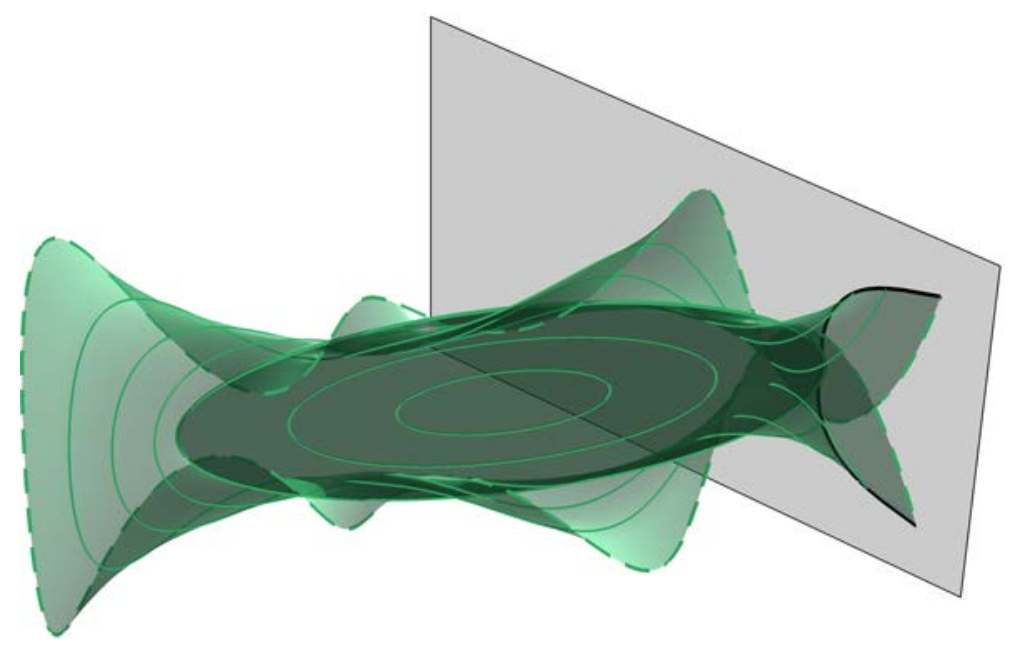

(a) Three-dimensional viewpoint

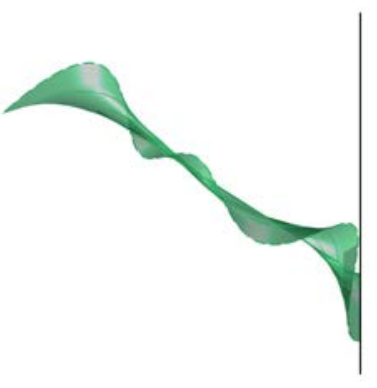

(b) Configuration space $\left(u_{N}, u_{N-1}\right)$ view

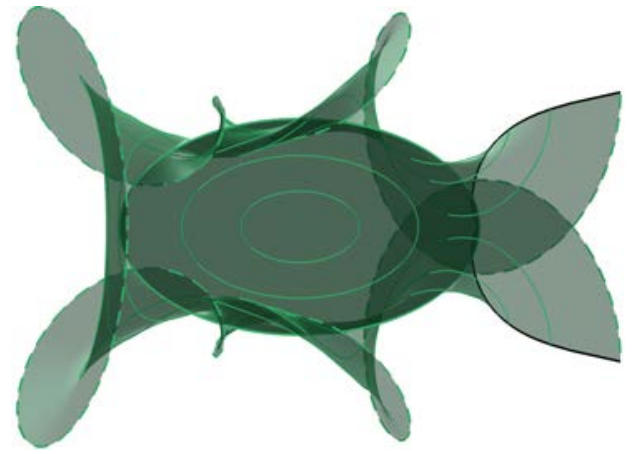

(c) Hyperplane $\left(u_{N-1}, \dot{u}_{N}\right)$ view

Figure 5.4: Nonsmooth mode for $T \approx T_{1}$ corresponding to branch 1 in Fig. 5.1

- the velocity:

$$
\begin{aligned}
& \dot{u}_{N}(t ; T)=\frac{g}{w_{N}(T)}\left(-\sum_{\ell=1}^{N} \sum_{k=1}^{N} P_{N \ell} P_{\ell k}^{-1} \omega_{\ell} \sin \left(\omega_{\ell} t\right) w_{k}(T)+\sum_{k=1}^{N} a_{k} \cos \left(\omega_{k} t\right)\right) \\
& \dot{u}_{N}^{+}(0 ; T)=\frac{g}{w_{N}(T)}\left(\sum_{k=1}^{N} a_{k}\right)=\frac{g}{w_{N}(T)},
\end{aligned}
$$

- the acceleration:

$$
\begin{aligned}
& \ddot{u}_{N}(t ; T)=-\frac{g}{w_{N}(T)}\left(\sum_{\ell=1}^{N} \sum_{k=1}^{N} P_{N \ell} P_{\ell k}^{-1} \omega_{\ell}^{2} \cos \left(\omega_{\ell} t\right) w_{k}(T)+\sum_{k=1}^{N} a_{k} \omega_{k} \sin \left(\omega_{k} t\right)\right) \\
& \ddot{u}_{N}^{+}(0 ; T)=-\frac{g}{w_{N}(T)}\left(\sum_{\ell=1}^{N} \sum_{k=1}^{N} P_{N \ell} P_{\ell k}^{-1} \omega_{\ell}^{2} w_{k}(T)\right)=-g \sum_{\ell=1}^{N} \sum_{k=1}^{N} P_{N \ell} P_{\ell k}^{-1} \omega_{\ell}^{2} \frac{w_{k}(T)}{w_{N}(T)}
\end{aligned}
$$

which becomes, by passing to the limit when $T \rightarrow T_{j}$

$$
\begin{aligned}
\lim _{T \rightarrow T_{j}} \ddot{u}_{N}^{+}(0 ; T) & =-g \sum_{\ell=1}^{N} \sum_{k=1}^{N} P_{N \ell} P_{\ell k}^{-1} \omega_{\ell}^{2} \frac{P_{k j}}{P_{N j}}=-g \sum_{\ell=1}^{N} \omega_{\ell}^{2} \frac{P_{N \ell}}{P_{N j}}\left(\sum_{k=1}^{N} P_{\ell k}^{-1} P_{k j}\right) \\
& =-g \sum_{\ell=1}^{N} \omega_{\ell}^{2} \frac{P_{N \ell}}{P_{N j}} \delta_{\ell j}=-g \omega_{j}^{2}<0 .
\end{aligned}
$$

To summarize, we can state that $u_{N}(0 ; T)=g, \dot{u}_{N}^{+}(0 ; T) \leq 0$ and $\ddot{u}_{N}^{+}(0 ; T)<0$ for $T \approx T_{j}$ and $a_{j}\left(T-T_{j}\right)<0$. Thus $u_{N}(t ; T)<g$ for $0<t<\epsilon, a_{j}\left(T-T_{j}\right)<0,\left|T-T_{j}\right|<\epsilon$ and $\epsilon>0$, which is sufficient to prove the admissibility of the solution. 


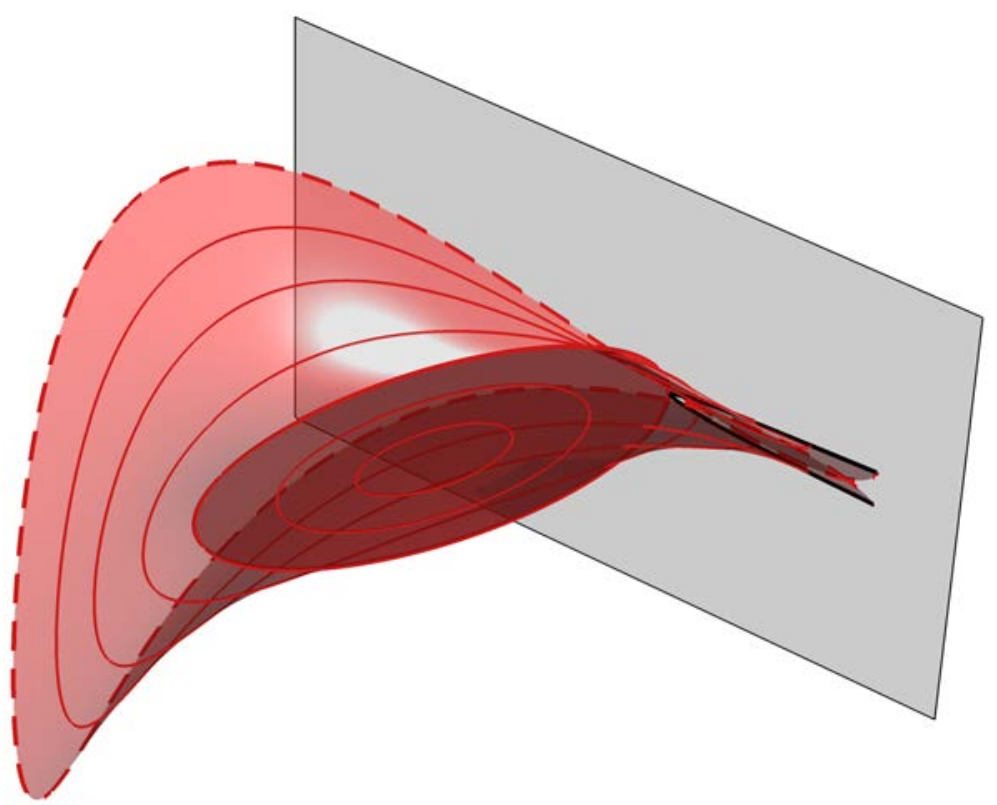

(a) Three-dimensional viewpoint

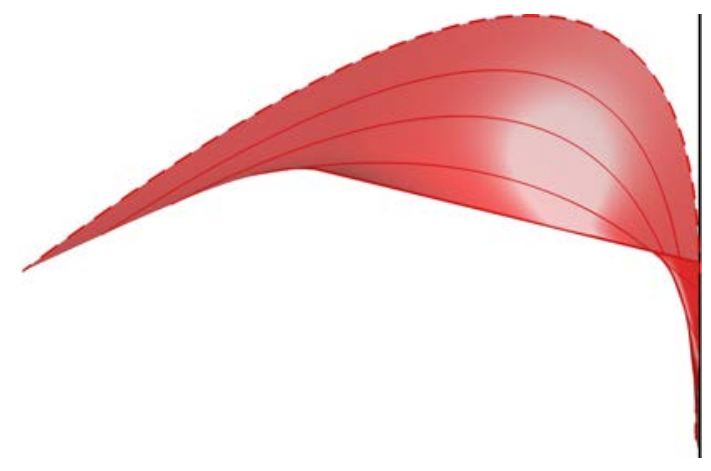

(b) Configuration space $\left(u_{N}, u_{N-1}\right)$ view

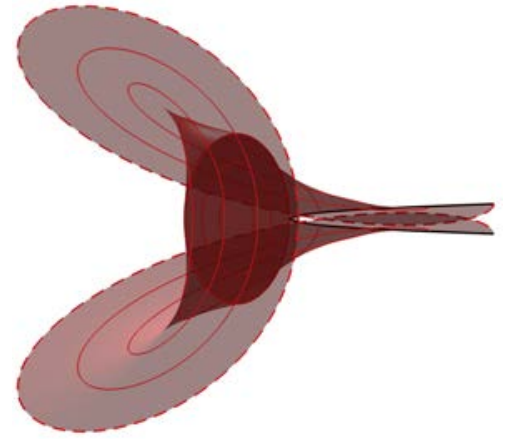

(c) Hyperplane $\left(u_{N-1}, \dot{u}_{N}\right)$ view

Figure 5.5: Nonsmooth mode for $T \approx T_{2}$ corresponding to branch 2 in Fig. 5.1

Two manifolds emerging in the neighborhood of the linear natural frequencies $\omega_{1}$ and $\omega_{2}$ are displayed in figures 5.4 and 5.5, respectively. They both exhibit a nonlinear grazing orbit limiting their domain of definition. Accordingly, these two manifolds have boundaries. A third one, unbounded, defined around $\omega_{3}$ is shown in Fig. 5.6. It should be compared to its counterpart arising about $\omega_{3} / 3$ : they both share the same linear portion but then bifurcate differently at the linear grazing orbit. A similar behavior is expected for all admissible subharmonics of all (linear) modes. As such, this is a typical example of a mechanical system for which the number of nonlinear modes of vibration exceeds the number of degrees-of-freedom.

5.2. In the vicinity of the linear subharmonics In this section, we prove Theorem 3.3 and discuss the more challenging case of linear subharmonics.

Proof. The solution $\mathbf{u}\left(t ; 2 T_{j}\right)$ is simply the $j$ th linear grazing solution with two loops and two (grazing) contacts per period. By continuity, it is clear that the solution $\mathbf{u}(t ; T) \simeq \mathbf{u}\left(t ; 2 T_{j}\right)$ when $T \approx 2 T_{j}$ (see Fig. 5.7). To test admissibility, attention is paid to the scalar function $t \mapsto u_{N}(t ; T)$ : from the closed-form expression (5.4), it clearly lies near $u_{N}\left(t ; 2 T_{j}\right)=g \cos \left(\omega_{j} t / 2\right)$ in all $C^{k}$ topologies (outside the impact). The solution $\mathbf{u}\left(t ; 2 T_{j}\right)$ and all its derivatives with respect to time $t$ have simple roots only, and this holds true for $T \approx 2 T_{j}$ as well. Accordingly, $\mathbf{u}(t ; T)$ has only two local maxima on $[0 ; T$ [ where the condition $\mathbf{u}(t ; T) \leq g$ has to be tested. If the one-sided condition $a_{j}\left(T-2 T_{j}\right)<0$ is satisfied for all $T \approx 2 T_{j}$, the first maximum is located at $t=0$ : this is a straightforward consequence of the previous proof. Due to the symmetry of the solution, the second maximum is at $t=T / 2$. We also have $\ddot{u}_{N}(T / 2 ; T) \sim \ddot{u}_{N}\left(T_{j} ; 2 T_{j}\right)=\ddot{u}_{N}\left(0 ; T_{j}\right)<0$ and the inequality $u_{N}(T / 2 ; T) \leq g$ guarantees admissibility.

As detailed in Proposition 4.1, interesting features of the modal dynamics can also be observed by examining responses for various initial energy levels in the phase portraits. Fig. 5.10 depicts phase plane 


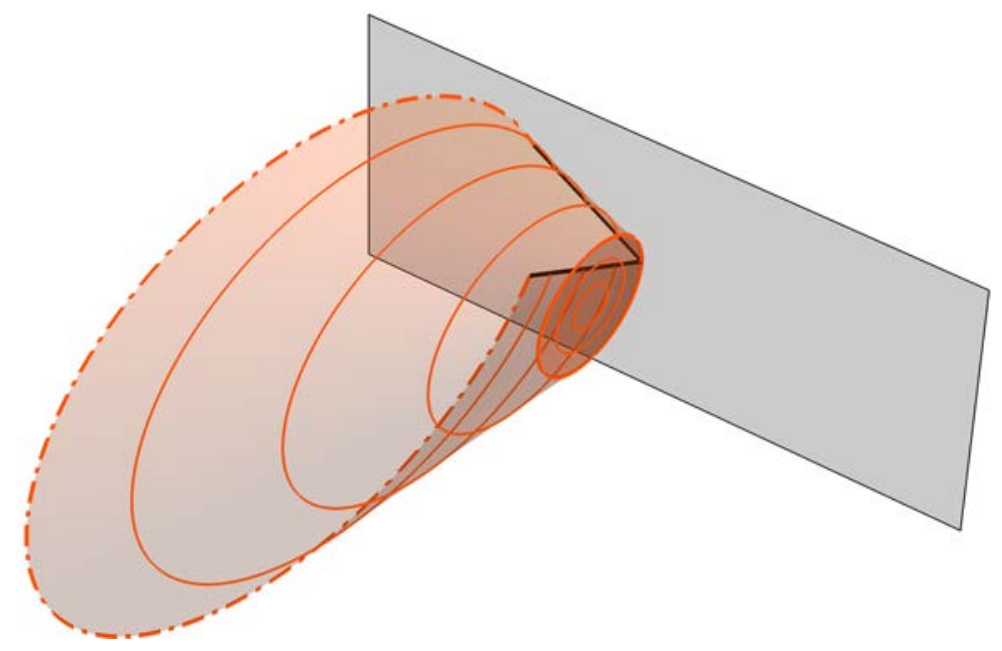

(a) Three-dimensional viewpoint

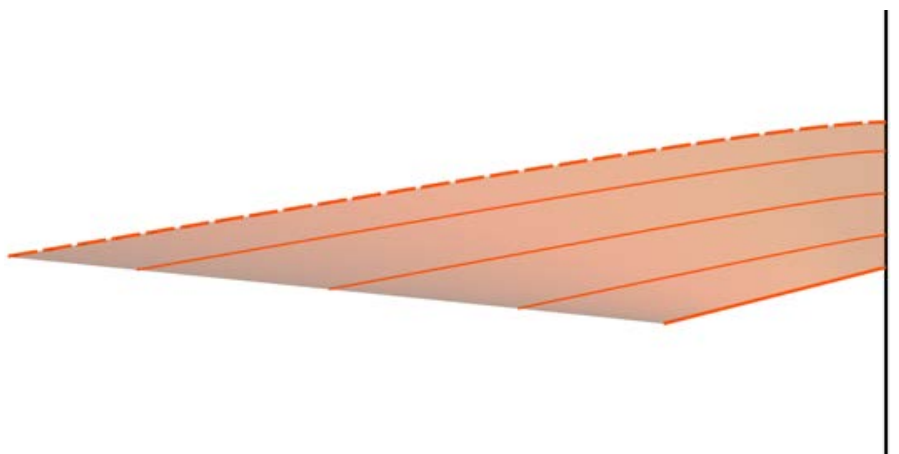

(b) Configuration space $\left(u_{N}, u_{N-1}\right)$ view

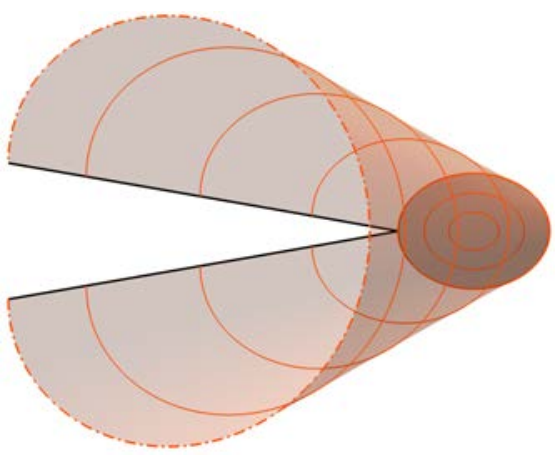

(c) Hyperplane $\left(u_{N-1}, \dot{u}_{N}\right)$ view

Figure 5.6: Nonsmooth mode for $T \approx T_{3}$. It is not bounded by a nonlinear grazing orbit. The corresponding branch is not shown in Fig. 5.1. It should be compared to the manifold emerging in the vicinity of $T \approx 3 T_{3}$ reported in Fig. 5.8. Both share the same linear part but bifurcate differently at the linear grazing orbit

diagrams for masses 4 and 5: the loops correspond to periodic responses whose amplitudes depend on the initial energy level. They are symmetric along the velocity axis because of the conservative nature of the system and the symmetry of the impulsive impact force in velocity. It also shows how mass 4 is affected in its motion by the nonlinear restitution force.

Remark 8. An important feature that distinguishes the investigated vibro-impact oscillator from polynomial nonlinear oscillators is the existence of physically observable backbone curves near the linear subharmonics of vibration. In Fig. 5.1, the vibratory motions corresponding to the two linear segments located at $\omega_{3} / 3$ and $\omega_{4} / 2$ are not readily observable as they turn to be identical to the ones corresponding to the two linear segments located at $\omega_{3}$ and $\omega_{4}$ (that is linear modes 3 and 4), respectively. Nevertheless, the nonlinear branches emanating from the natural frequencies $\omega_{i}$ and from their subharmonics $\omega_{i} / j$, $(i, j) \in \mathbb{N}^{*+}$, correspond to distinct co-existing manifolds sharing the same linear portion, as illustrated in Figs. 5.6 and 5.8. This is in contrast with some polynomial nonlinear systems where manifolds are defined in the neighborhood of linear modes of vibration only. In other words, a countable set of manifolds (potentially infinitely many and each of them defining a nonlinear mode of vibration) emerge from the linear fundamental grazing orbits, and the Poincaré map is thus singular.

Admissibility for $T \simeq k T_{j}, k>2$ For lower-order subharmonics $(1 / 3,1 / 4, \ldots)$, numerical approximations are required to test admissibility conditions:

1. Check $a_{j}\left(T-k T_{j}\right)<0$.

2. With a numerical solver, find $t_{\ell} \approx \ell T / k$ such that $\dot{u}_{N}\left(t_{\ell} ; T\right)=0$ for all integers $\ell$ such that $1 \leq \ell \leq k / 2$.

3. Check the conditions $u_{N}\left(t_{\ell} ; T\right) \leq g$ for $1 \leq \ell \leq k / 2$.

As shown in Fig. 5.7, the displacement $u_{N}(\cdot ; T)$ on $[0 ; T]$ has a graph very similar to $u_{N}\left(\cdot ; k T_{j}\right)$ on 
$\left[0 ; k T_{j}\right]$ with the same number of extrema nearly at the same locations. By symmetry, only half a period should be scrutinized. Moreover, if $k$ is even, $t_{k / 2}=T / 2$ as in Theorem 3.3.

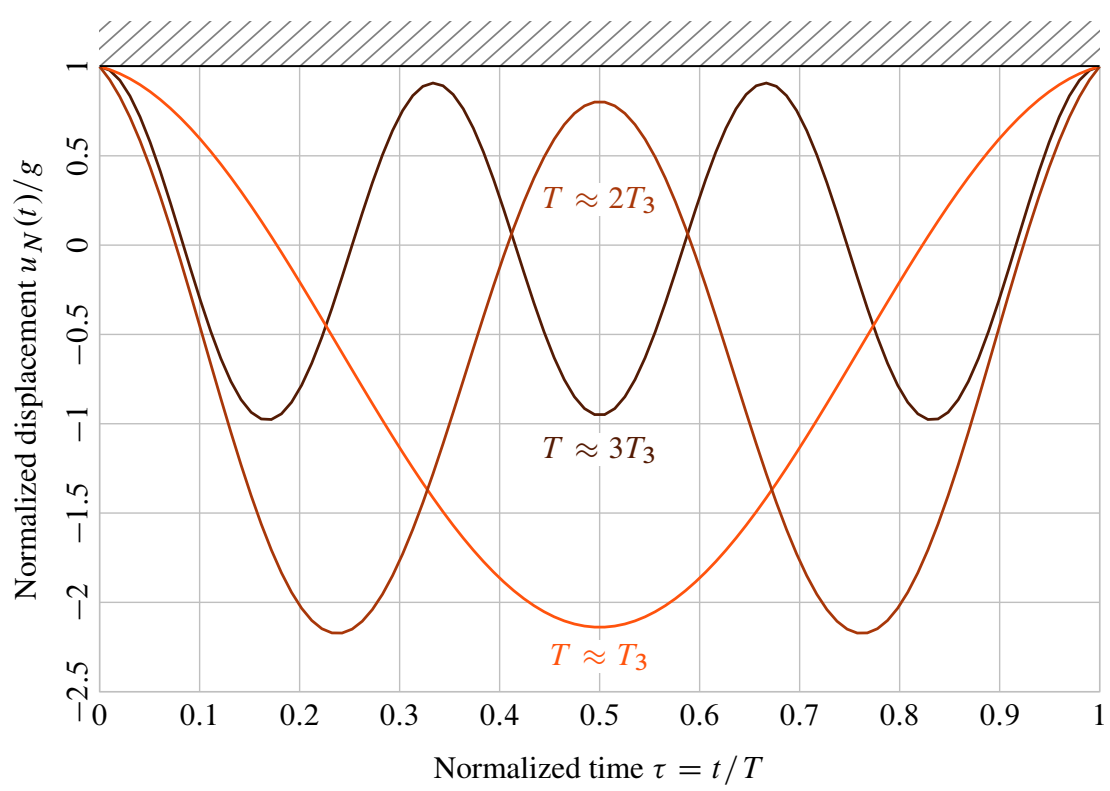

Figure 5.7: Admissible nonsmooth periodic solutions in the vicinity of the third linear mode of period $T_{3}$, and in the vicinity of two of its subharmonics $2 T_{3}$ and $3 T_{3}$. For readability, the time scale is normalized with respect to each $T$

5.3. High Frequencies In [23, p. 1008], it is shown that very high frequency (that is $T \rightarrow 0$ here) admissible solutions exist. This is not true in our investigation. The last statement of Theorem 3.2 is a consequence of Proposition 5.2.

Proposition 5.2 [Nonexistence of high-frequency nonlinear modes] If $\alpha:=\sum_{j=1}^{N} a_{j} \omega_{j}^{-2}>0$ then the frequencies of the admissible nonlinear modes are bounded.

As previously highlighted, our illustrative example (2.17) is such that the interaction coefficients are all positive and the admissible frequencies are bounded. In order to observe high-frequency admissible nonlinear modes, the condition $\alpha<0$ is required which is a bit challenging to fulfill since $\sum_{j} a_{j}=1$ from (2.12).

Proof. Within the current mathematical framework, it is possible to derive an asymptotic behaviour when $T \rightarrow 0$. The limit $\lim _{T \rightarrow 0} \mathbf{u}(0 ; T)$ can be explicitly exploited and the only non vanishing velocity $\dot{u}_{N}^{+}(0 ; T)=$ $g / w_{N}(T)=\mathcal{O}(T)$ converges to 0 . Recall that $u_{k}(0 ; T)=g w_{k}(T) / w_{N}(T)$. The asymptotic behavior of $w_{k}(T)$ for $T \approx 0$ is

$$
w_{k}(T)=\sum_{p=1}^{n} P_{k p} P_{p N}^{-1} \phi_{p}(T)=\frac{2}{T} \sum_{p=1}^{N} P_{k p} P_{p N}^{-1} \omega_{p}^{-2}+\mathcal{O}(1) \text { when } T \rightarrow 0 .
$$

In particular, for $k=N$, we simply have $w_{N}(T) \sim 2 \alpha / T$ when $T \rightarrow 0$. Consequently, the limiting initial data are

$$
\begin{array}{ll}
\lim _{T \rightarrow 0} u_{k}(0 ; T)=\frac{g}{\alpha} \sum_{p=1}^{N} P_{k p} P_{p N}^{-1} \omega_{p}^{-2}, & k=1, \ldots, N \\
\lim _{T \rightarrow 0} \dot{u}_{k}(0 ; T)=0, & k=1, \ldots, N .
\end{array}
$$

In particular, $u_{N}(0 ; 0)=g$ and a grazing contact trajectory is retrieved. The admissibility criterion on the velocity requires $\dot{u}_{N}(0 ; T)<0$ but for $T \gtrsim 0, \dot{u}_{N}^{+}(0 ; T)=g / w_{N}(T) \sim T g /(2 \alpha)>0$. Thus the solution of the extended formulation (Definition 2.1) becomes inadmissible. This is sufficient to conclude the proof. Nevertheless, the second derivative in time is also computed as it is later used in the pre-stressed case. Through 


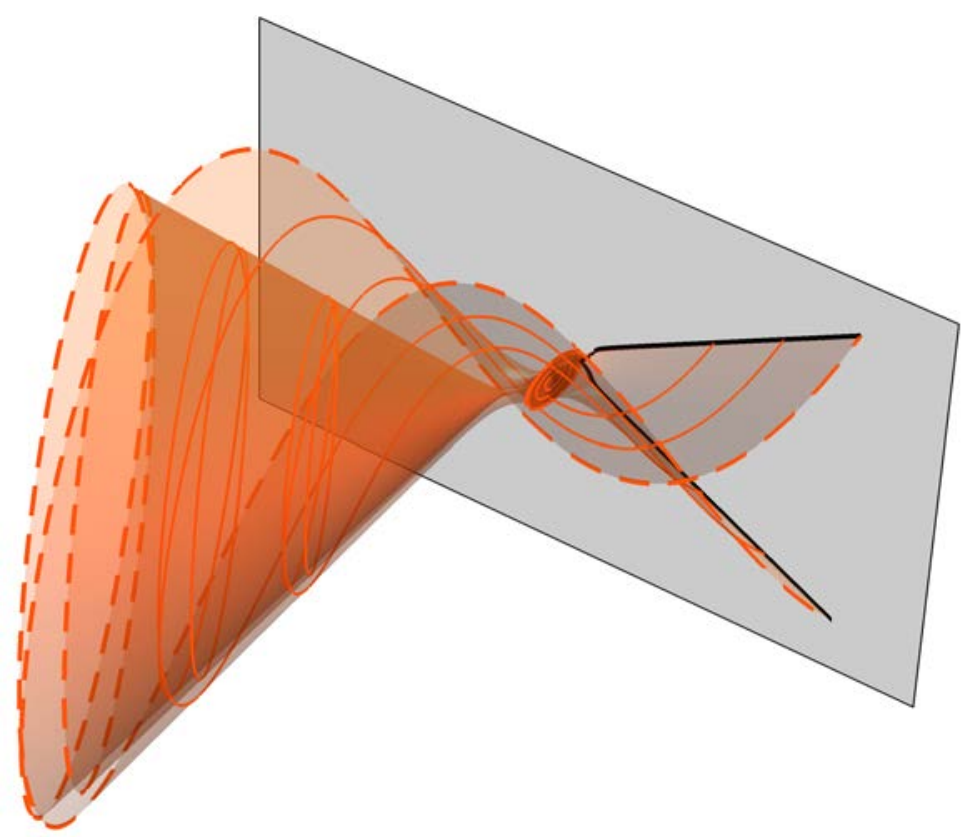

(a) Three-dimensional viewpoint

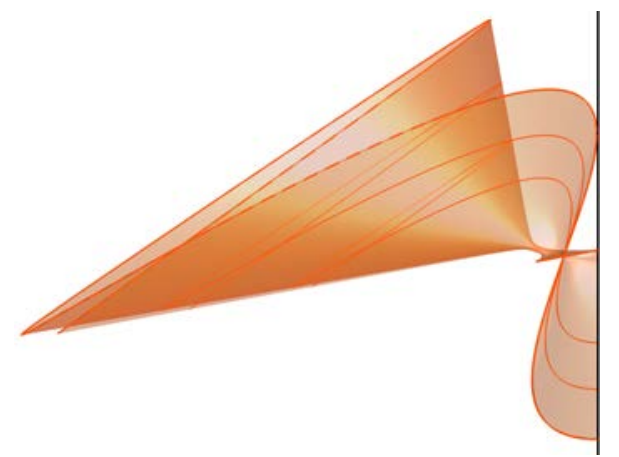

(b) Configuration space $\left(u_{N}, u_{N-1}\right)$ view

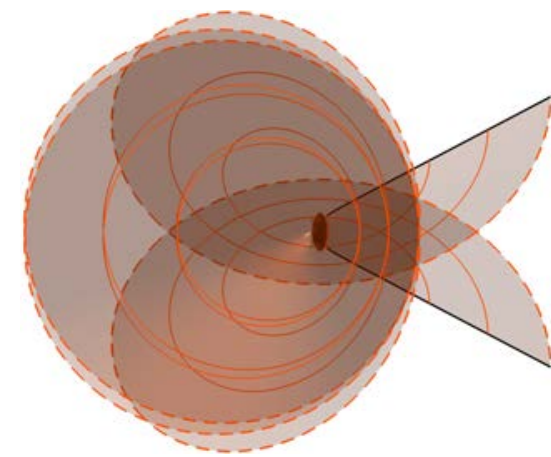

(c) Hyperplane $\left(u_{N-1}, \dot{u}_{N}\right)$ view

Figure 5.8: Nonsmooth mode for $T \approx 3 T_{3}$ corresponding to branch 3 in Fig. 5.1

Eqs. (5.10) and (5.3), we can derive an approximation of the second right derivative for small $T$ :

$$
\begin{aligned}
\ddot{u}_{N}^{+}(0 ; T) & =-g \sum_{\ell=1}^{N} \sum_{k=1}^{N} P_{N \ell} P_{\ell k}^{-1} \omega_{\ell}^{2} \frac{w_{k}(T)}{w_{N}(T)} \approx-\frac{g}{\alpha} \sum_{\ell=1}^{N} \sum_{k=1}^{N} \sum_{p=1}^{N} \frac{\omega_{\ell}^{2}}{\omega_{p}^{2}} P_{N \ell} P_{\ell k}^{-1} P_{k p} P_{p N}^{-1} \\
& \approx-\frac{g}{\alpha} \sum_{\ell=1}^{N} \sum_{p=1}^{N} \frac{\omega_{\ell}^{2}}{\omega_{p}^{2}} P_{N \ell} P_{p N}^{-1} \delta_{\ell p}=-\frac{g}{\alpha} \sum_{\ell=1}^{N} P_{N \ell} P_{\ell N}^{-1} \sim-\frac{g}{\alpha}<0 .
\end{aligned}
$$

Accordingly, the trajectory leaves the rigid foundation on the "wrong" non-admissible side $u_{N}>g$ with decreasing velocity.

6. Spectral stability analysis Nonlinear systems shall exhibit a wide range of exotic behaviors, including instabilities, bifurcations, and chaos. Of high interest is the systematic stability analysis of the previously found periodic orbits as their frequency—or energy—increases. Stability of a periodic solution manifests itself in the way neighboring trajectories behave. It is known for autonomous conservative linear systems that the periodic orbits are neutrally stable. In general, this property is destroyed for nonlinear systems and as a result, some of the previously found orbits shall be unstable. In order to clarify the situation, a dedicated analysis should be undertaken: it commonly prescribes a perturbation of a calculated periodic trajectory in order to explore its dynamical behavior with time. This can be conveniently achieved by either calculating the eigenvalues of the linearized Poincaré map or, equivalently, by exploiting the characteristic multipliers determined from the monodromy matrix [25].

In this work, since the First Return Map is found to be locally well defined and smooth for non-grazing 


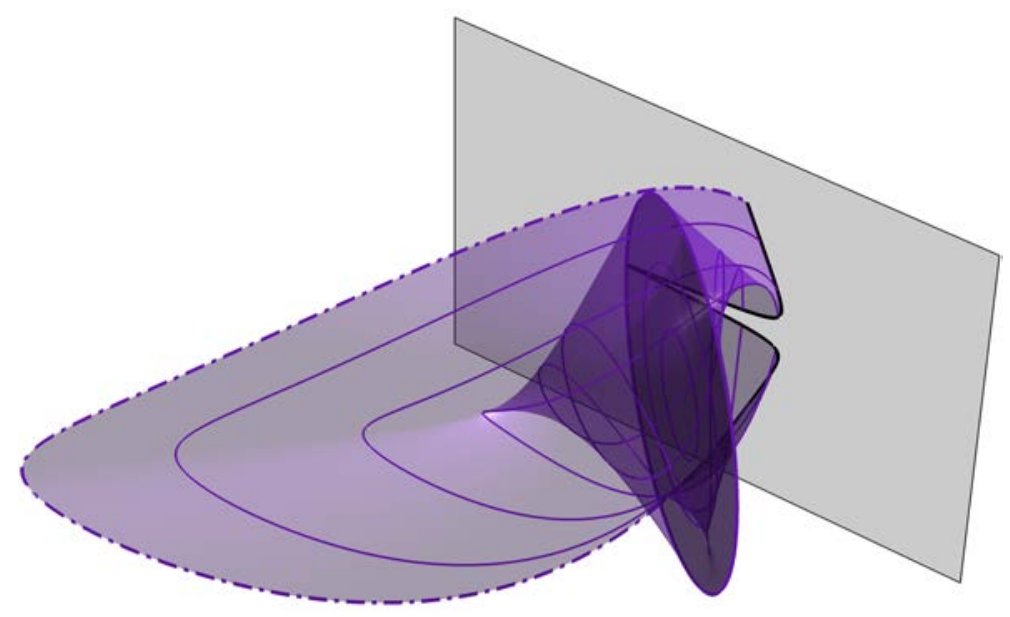

(a) Three-dimensional viewpoint

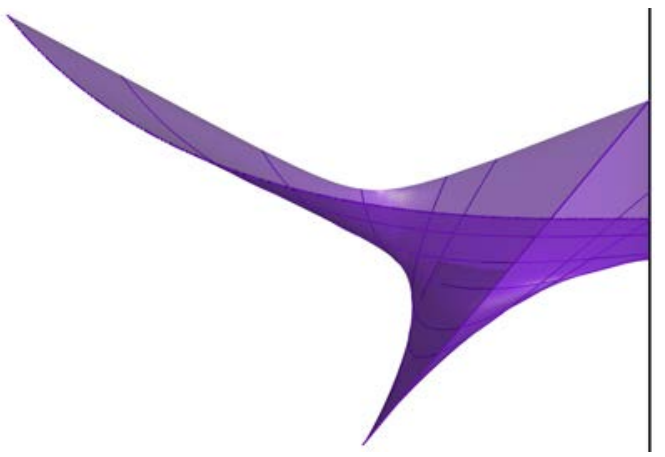

(b) Configuration space $\left(u_{N}, u_{N-1}\right)$ view

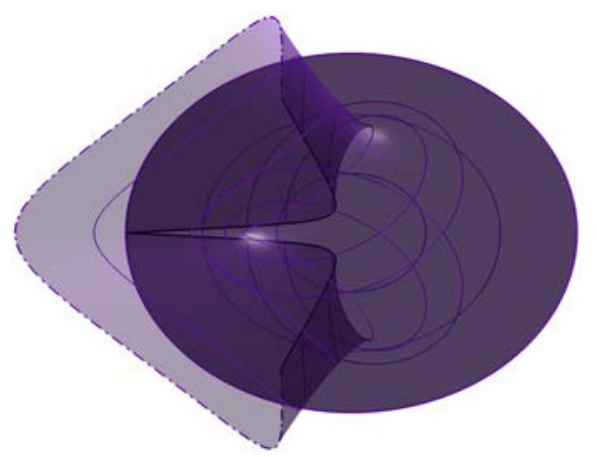

(c) Hyperplane $\left(u_{N-1}, \dot{u}_{N}\right)$ view

Figure 5.9: Nonsmooth mode for $T \approx 2 T_{4}$ corresponding to branch 4 in Fig. 5.1

solutions ${ }^{5}$, Lyapunov's theorems can be used and the stability analysis reduces to the classical spectral stability analysis of the Poincare map fixed points [10]. The sufficient condition for (spectral) stability is that all the eigenvalues of the linearized Poincaré map lie inside the unit circle in the complex plane; the necessary condition is that they lie either inside or on the circle. In other words, instability is achieved as soon as the modulus of one eigenvalue of the linearized First Return Map exceeds unity.

Additional features of the response are also readily accessible from this analysis: depending on where the eigenvalue or pair of complex conjugate eigenvalues crosses the unit circle defined in the complex plane, different types of bifurcation occur. Evidently, the more challenging bifurcation mechanisms induced by grazing orbits, that are specific to nonlinear systems involving switching manifolds in the state-space, are not addressed with this approach.

6.1. Linearized First Return Map In order to prove Corollary 3.1, let us first notice that the First Return Map $\mathbf{F}$ is fully defined by the first return time $T$ when it exists. Also, we need the following ingredient: the mechanical energy $E(\dot{\mathbf{u}}, \mathbf{u})=\dot{\mathbf{u}}^{\top} \mathbf{M} \dot{\mathbf{u}}+\mathbf{u}^{\top} \mathbf{K u}$ is conserved after an impact when the last mass is concentrated.

Proposition 6.1 [Conserved energy] The impact law conserves the mechanical energy if and only if the mass matrix of the investigated system satisfies $M_{N k}=0=M_{k N}$ for all $k=1, \ldots, N-1$.

Proof. In space coordinates, the energy is clearly conserved during the free flight. The impact rule only affects velocities since it reads $\dot{\mathbf{u}}^{+}=\mathbf{S} \dot{\mathbf{u}}^{-}$. The energy is conserved only if the kinetic energy is preserved during an impact, thus $\mathbf{S M S}=\mathbf{M}$, i.e. $-M_{N k}=M_{N k}$ and $-M_{k N}=M_{k N}$ for all $k=1, \ldots, N-1$.

Corollary 3.1 is now proven. Its relevance lies in the explicit formula to compute Floquet's exponents. The existence and the computation of the linearized First Return Map are well known [7] since there

5 The stability analysis of the grazing orbits involves quite complicated arguments $[4,13]$ that are not discussed in this paper. 


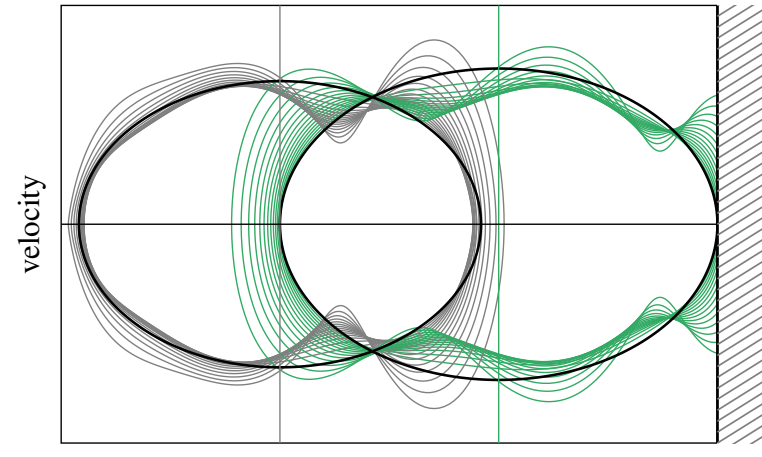

displacement [+ initial position]

(a) Branch 1

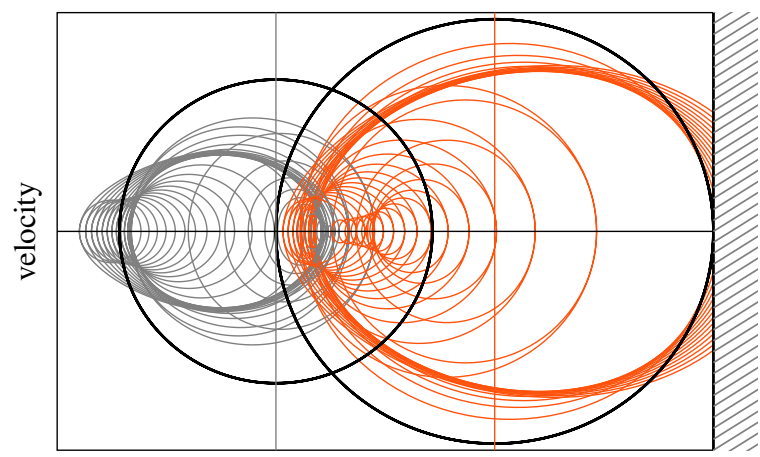

displacement [+ initial position]

(c) Branch 3

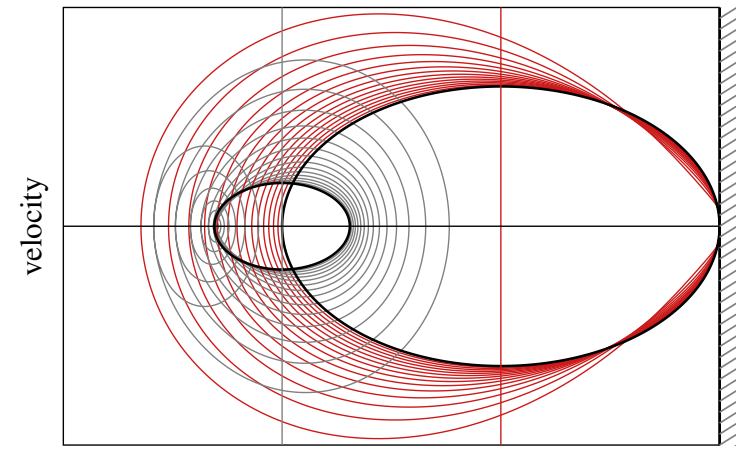

displacement [+ initial position]

(b) Branch 2

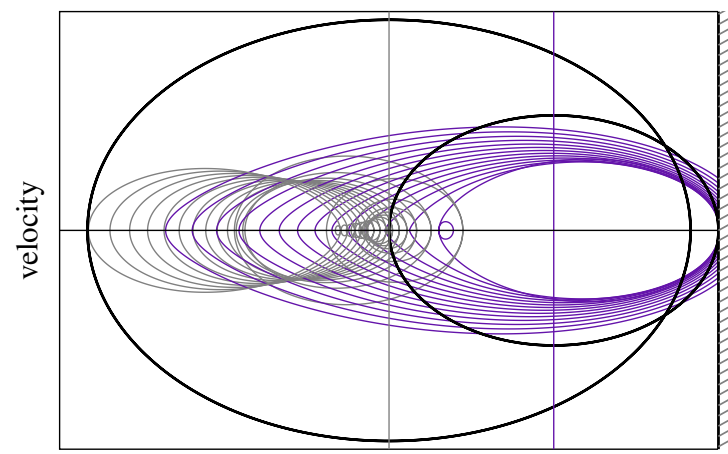

displacement [+ initial position]

(d) Branch 4

Figure 5.10: Phase portrait of the periodic motion on the invariant manifolds: mass $N$ [color] and mass $N-1$ [gray]. Linear grazing orbit [thick black]. Branches are defined in Fig. 5.1

is no grazing contact. To be self-contained, the proof and computation are proposed using our closedform solutions. Derivations more in line with the Mechanical Engineering community are provided in Appendix A.

Proof. To avoid the impact-induced jump in the orbit, the closed-form solution

$$
\mathbf{q}(t)=\mathbf{L}(t) \mathbf{Q}=\cos (t \boldsymbol{\Omega}) \mathbf{q}(0)+\mathbf{\Omega}^{-1} \sin (t \boldsymbol{\Omega}) \dot{\mathbf{q}}^{+}(0)
$$

is considered on interval ] $0 ; T$ [ as a function of initial data $\mathbf{Q}$ and time $t$ where the $N \times 2 N$ matrix $\mathbf{L}(t)$ stores the first $N$ rows of $\mathbf{R}(t)$. The linear relation (6.1) is valid only on [0;T]. Away from impact, $\mathbf{q}(t)$ can be differentiated with respect to time such that $\dot{\mathbf{q}}(t)=\dot{\mathbf{L}}(t) \mathbf{Q}$ while at the impact, $\dot{\mathbf{q}}^{-}(T)=\dot{\mathbf{L}}(T) \mathbf{Q}$.

The proof involves several distinct steps:

1. Existence and smoothness of $T$ in the neighborhood of $\mathbf{Q}_{0}-$ The scalar function $T(\mathbf{Q})$ is implicitly defined by $u_{N}(T(\mathbf{Q}))=g$ in physical coordinates which becomes, in modal coordinates

$$
f(T, \mathbf{Q}):=\mathbf{e}_{N}^{\top} \mathbf{P L}(T) \mathbf{Q}
$$

Note also that $f(T, \mathbf{Q})=\overline{\mathbf{e}}_{N}^{\top} \overline{\mathbf{P}} \mathbf{R}(T) \mathbf{Q}$. In order to use the implicit function theorem at $\left(T_{0}, \mathbf{Q}_{0}\right)$, it is sufficient to prove that $\partial_{T} f\left(T_{0}, \mathbf{Q}_{0}\right) \neq 0$. This partial derivative has a simple interpretation: $\partial_{T} f\left(T_{0}, \mathbf{Q}_{0}\right)=\dot{u}_{N}^{-}\left(T_{0} ; T_{0}\right)$ since $u_{N}\left(t ; T_{0}\right)=f\left(t, \mathbf{Q}_{0}\right)$ for all $\left.t \in\right] 0 ; T_{0}\left[\right.$. Accordingly, the non-grazing condition $\dot{u}_{N}^{+}\left(\left(0 ; T_{0}\right)\right) \neq 0$, the periodicity, and the reflection law $\dot{u}_{N}^{-}\left(T_{0} ; T_{0}\right)=-\dot{u}_{N}^{+}\left(T_{0} ; T_{0}\right)=-\dot{u}_{N}^{+}\left(0 ; T_{0}\right)$ show that

$$
\partial_{T} f\left(T_{0}, \mathbf{Q}_{0}\right)=\mathbf{e}_{N}^{\top} \mathbf{P} \dot{\mathbf{L}}\left(T_{0}\right) \mathbf{Q}=-\dot{u}_{N}^{+}\left(0 ; T_{0}\right) \neq 0 .
$$

2. Explicit formula for $\mathbf{D F}$ - Differentiating equality (6.2) with respect to $\mathbf{Q}$ at $\mathbf{Q}=\mathbf{Q}_{0}$ leads to

$$
\begin{aligned}
\nabla T & =-\frac{1}{\partial_{T} f} \nabla_{\mathbf{Q}} f=-\frac{1}{\mathbf{e}_{N}^{\top} \mathbf{P} \dot{\mathbf{L}}\left(T\left(\mathbf{Q}_{0}\right)\right) \mathbf{Q}_{0}} \mathbf{e}_{N}^{\top} \mathbf{P L}\left(T\left(\mathbf{Q}_{0}\right)\right)=\frac{1}{\dot{u}_{N}^{+}\left(0 ; T_{0}\right)} \mathbf{e}_{N}^{\top} \mathbf{P L}\left(T_{0}\right) \\
& =\frac{w_{N}\left(T_{0}\right)}{g} \mathbf{e}_{N}^{\top} \mathbf{P L}\left(T_{0}\right)=\frac{w_{N}\left(T_{0}\right)}{g} \overline{\mathbf{e}_{N}^{\top}} \overline{\mathbf{P}} \mathbf{R}\left(T_{0}\right) .
\end{aligned}
$$

Finally, it is sufficient to differentiate $\mathbf{F}$ through the standard chain rule formula. 
3. 1 is an eigenvalue - There are two ways to see that 1 is an eigenvalue of DF. First, we have a smooth family of initial data $\mathbf{Q}(0 ; T)$ for a non-grazing nonsmooth mode, so $\mathbf{F}(\mathbf{Q}(0 ; T))=\mathbf{Q}(0 ; T)$ and differentiating with respect to the time yields

$$
\mathbf{D F}(\mathbf{Q}(0 ; T)) \partial_{T} \mathbf{Q}(0 ; T)=\partial_{T} \mathbf{Q}(0 ; T) .
$$

The explicit formula for $\mathbf{Q}(0 ; T)$ shows that $\partial_{T} \mathbf{Q}(0 ; T) \neq \mathbf{0}$ at least for almost all $T$. In this case $\partial_{T} \mathbf{Q}(0 ; T)$ is a left eigenvector of $\mathbf{D F}$ associated with the eigenvalue $1^{6}$.

Second, if the mass matrix satisfies the assumptions of Proposition 6.1, differentiating the conservation of energy $E(\mathbf{F}(\mathbf{Q}))=E(\mathbf{Q})$ yields $\nabla E(\mathbf{F}(\mathbf{Q})) \mathbf{D F}(\mathbf{F}(\mathbf{Q}))=\nabla E(\mathbf{Q})$. Note that the energy is not degenerate, that is $\nabla E(\mathbf{Q}) \neq \mathbf{0}$ except for $\mathbf{Q}=\mathbf{0}$ which is not an initial condition for periodic solutions. Furthermore, if $\mathbf{F}(\mathbf{Q})=\mathbf{Q}$ then $\nabla E(\mathbf{Q})$ is a left eigenvector of $\mathbf{D F}(\mathbf{Q})$ with eigenvalue 1. We cannot deduce that 1 is an eigenvalue with multiplicity 2 as it is for smooth Hamiltonian systems.

The First Return Map is conventionally defined on the Poincare section only, that is the half hyperplane $\mathrm{H}^{+}$in this work. In the derivations above, the First Return Map and its differential in the neighborhood of an admissible nonlinear trajectory are expressed in the entire state-space, which adds one dimension, and thus one eigenvalue to the Jacobian. An obvious question then arises: "What is the magnitude and meaning of the additional eigenvalue?" Consider the orbit of the nonlinear mode which leaves $H^{+}$at time $t=0^{+}$: $\mathbf{F}\left(\mathbf{Q}\left(t ; T_{0}\right)\right)=\mathbf{Q}\left(0 ; T_{0}\right)=\mathbf{Q}_{0}$ such that $\mathbf{D F}\left(\mathbf{Q}_{0}\right)$ satisfies $\mathbf{D F}\left(\mathbf{Q}_{0}\right) \mathbf{v}=\mathbf{0}$ where $\mathbf{v}=\dot{\mathbf{Q}}\left(0 ; T_{0}\right) \neq \mathbf{0}$ does not belong to $H$. Consequently, in this formulation, where the unnecessary new dimension makes it easier to handle, 0 is always an extraneous eigenvalue of the matrix $\mathbf{D F}\left(\mathbf{Q}_{0}\right)$, that should be formally removed in the analysis.

6.2. Numerical example: first nonlinear mode Computational work can be directly conducted in physical coordinates where the $N$ th row of $\mathbf{D F}$ vanishes since $\mathbf{e}_{N}^{\top} \mathbf{D F}=\mathbf{0}$ which is a direct consequence of $\mathbf{F}(H) \subset H$. The varied control parameter is the frequency of the autonomous periodic response. The corresponding stability/instability pattern is shown in Fig. 6.1. As depicted in Fig. 6.2, instability arises through the three well-known bifurcation scenarios usually identified for autonomous and conservative systems: a pair of complex conjugate eigenvalues leaves the unit circle, defined in the complex plane, either (i) at $(1,0)$, or (ii) at $(-1,0)$, or (iii) anywhere else. For each of the above scenarios, eigenvalues initially travel on the unit circle and escape it, for critical values of the control parameter, yielding unstable behavior. Some of the additional features of the bifurcated solutions (fold, flip or Neimark-Sacker bifurcations, for instance) can be graphically displayed by numerically integrating the equations of motion initiated on the nonlinear mode. The Poincaré map is then employed to explore and visualize the dynamics in the unstable region. This is not further discussed in this work.

7. Pre-stressed configurations The initially open clearance $g$ is now modified as follows:

- A strictly pre-stressed configuration where the oscillator is statically distorted at rest (pre-stressed in Figure 7.1), that is $g<0$. All solutions are solely nonlinear with impacts. As shown later, the nonlinear spectrum is continuous but not connected to the linear spectrum, as it is for $g>0$.

- A closed-gap configuration where the system grazes the rigid foundation at rest (closed gap in Figure 7.1) that is $g=0$. The spectrum is discrete but distinct from the linear spectrum.

Structures of these invariant manifolds are presented.

7.1. Strict pre-stress The previous explicit closed forms for $g>0$ are still valid for $g<0$. Let us briefly recall and parametrize them with respect to $g$. The unique solution $\mathbf{u}(t ; T, g)$ to Definition 2.1 with $g \neq 0$, period $T>0$, and one impact per period such that $0<\left|w_{N}(T)\right|<\infty$ has the initial data

$$
\left(\begin{array}{c}
\mathbf{u}(0 ; T, g) \\
\dot{\mathbf{u}}^{+}(0 ; T, g)
\end{array}\right)=\frac{g}{w_{N}(T)}\left(\begin{array}{c}
\mathbf{w}(T) \\
\mathbf{e}_{N}
\end{array}\right)
$$

The poles of $w_{N}(\cdot)$ are exactly the periods (fundamental and multiple) of the linear differential system (2.6a). Periods $T$ such that $w_{N}(T)=0$ correspond to infinite energy and are forbidden for $g \neq 0$

\footnotetext{
$6 \longdiv { \text { The derivative } \partial _ { T } \mathbf { Q } ( 0 ; T ) \text { can eventually be } \mathbf { 0 } \text { for an isolated time only. Thus, for almost all time, this vector is non-zero and } 1 }$ is an eigenvalue. Since the eigenvalue depends continuously on the matrix coefficients that are smooth with respect to $T, 1$ is an eigenvalue for all time.
} 

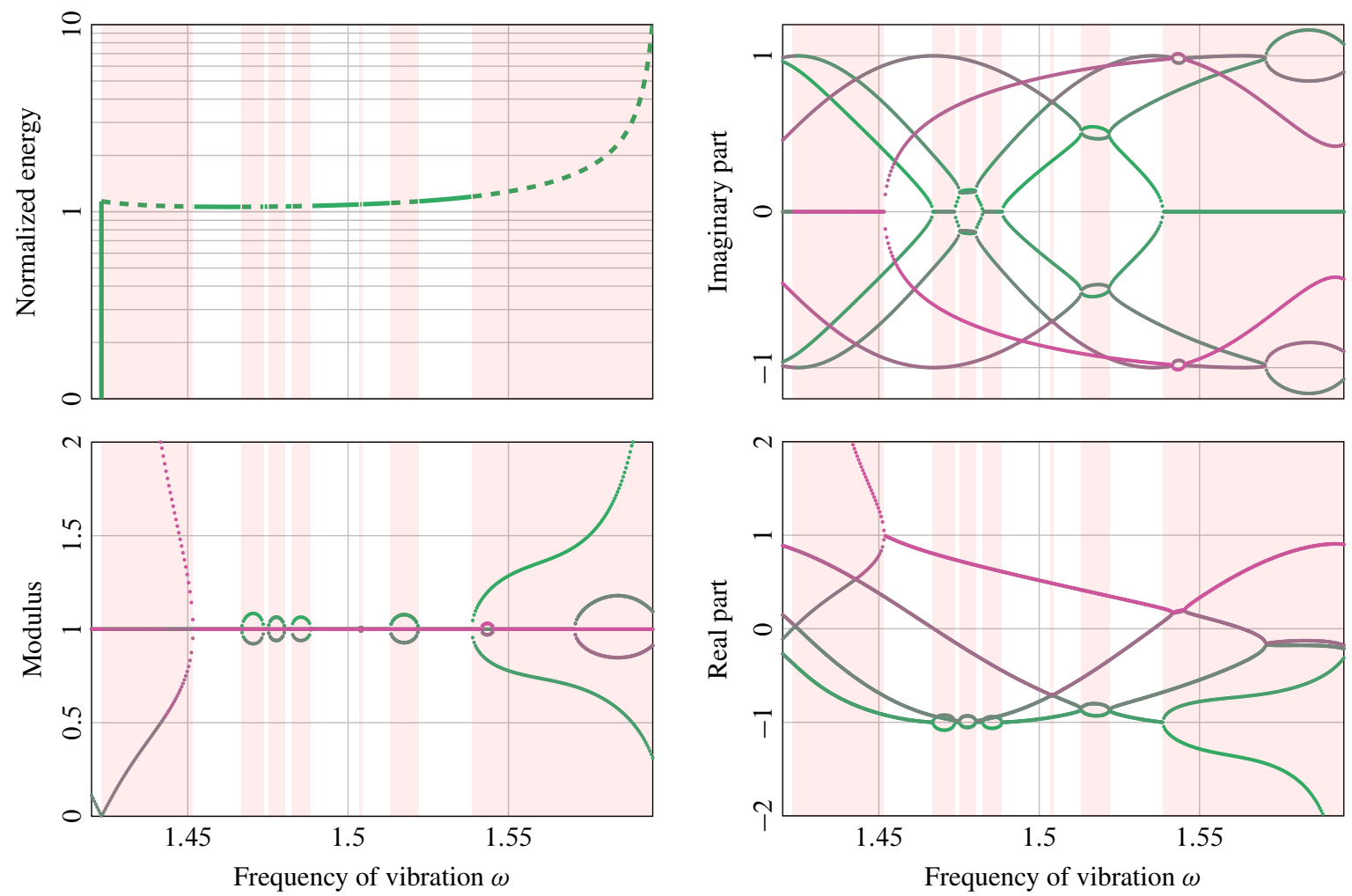

Figure 6.1: Spectral stability analysis of the first nonlinear mode in the vicinity of $\omega_{1}$ [Branch 1 in Fig. 5.1]. Instability is achieved as soon as the modulus of one eigenvalue of the linearized First Return Map DF is larger than unity. In every subfigure, zones of instability are highlighted in light red rectangles. The color scheme based on the magnitude of the real parts allows for the convenient identification of the various branches. Eigenvalue 1 is removed for readability purposes

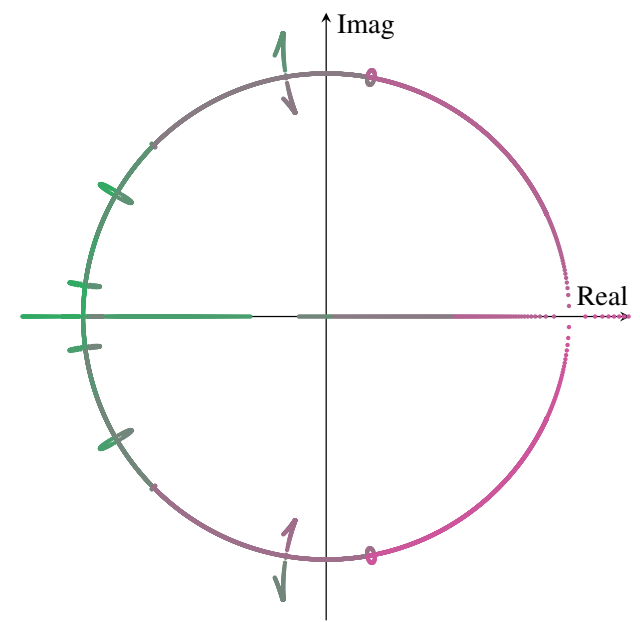

Figure 6.2: Spectral stability analysis of the first nonlinear mode in the vicinity of $\omega_{1}$ [Branch 1 in Fig. 5.1].

Eigenvalues of the linearized First Return Map DF in the complex plane

but allowed for $g=0$. The closed-form solutions are simply (see (5.4) and (5.5)):

$$
\mathbf{u}(t ; T, g)=\frac{g}{w_{N}(T)} \mathbf{P}\left(\cos (t \boldsymbol{\Omega}) \boldsymbol{\Phi}(T)+\boldsymbol{\Omega}^{-1} \sin (t \boldsymbol{\Omega})\right) \mathbf{P}^{-1} \mathbf{e}_{N}
$$

whose last component is, with $P_{\ell k}^{-1}=\left(\mathbf{P}^{-1}\right)_{\ell k}$ :

$$
u_{N}(t ; T, g)=\frac{g}{w_{N}(T)}\left(\sum_{\ell=1}^{N} \sum_{k=1}^{N} P_{N \ell} P_{\ell k}^{-1} \cos \left(\omega_{\ell} t\right) w_{k}(T)+\sum_{k=1}^{N} a_{k} \frac{\sin \left(\omega_{k} t\right)}{\omega_{k}}\right) .
$$

A new static equilibrium denoted $\mathbf{p}=\left(p_{1}, \ldots, p_{N}\right)$ and induced by pre-stress is reached: by definition, $p_{N}=g$ and the only unknown is $\widetilde{\mathbf{p}}=\left(p_{1}, \ldots, p_{N-1}\right)$ solution to the following $(N-1) \times$ 


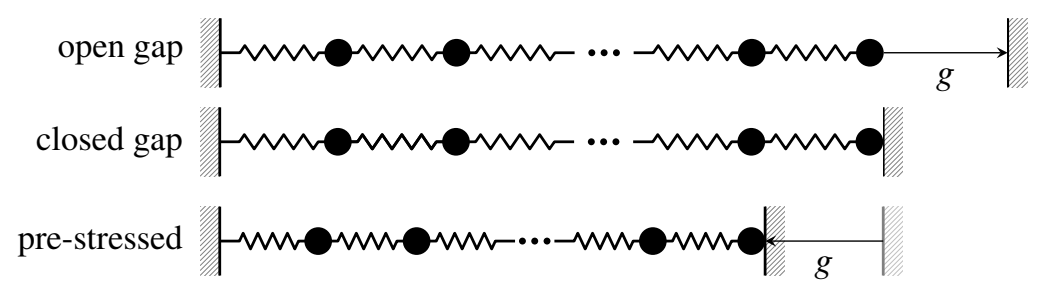

Figure 7.1: The three configurations of interest at rest

$(N-1)$ system with $1 \leq i, j \leq N-1$ :

$$
\widetilde{\mathbf{K}} \widetilde{\mathbf{p}}=\widetilde{\mathbf{b}}, \quad \widetilde{K}_{i j}=K_{i j}, \quad \widetilde{b}_{i}=-K_{i N} g .
$$

From Proposition 7.1 below, the pre-stressed static equilibrium p provides an upper bound on the frequencies of vibration.

The closed forms (7.1) and (7.2) are still valid when $g<0$. Then, the question of admissibility remains, namely to satisfy constraint (2.5c). Numerical examples are provided in Figures 7.2(a) and 7.2(b) where two portions of the admissible manifold are shown.

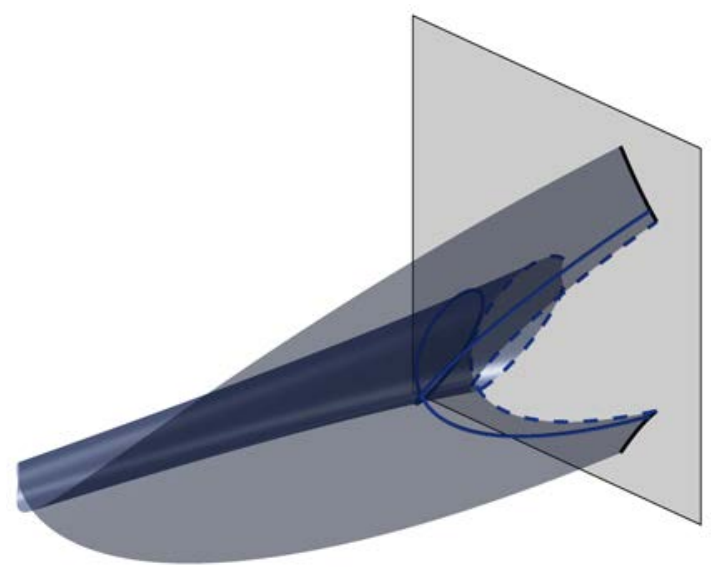

(a) $\omega_{\mathrm{n} 13}<\omega \leq \omega_{\mathrm{n} 11}$ (branch 1 in Fig. 7.3)

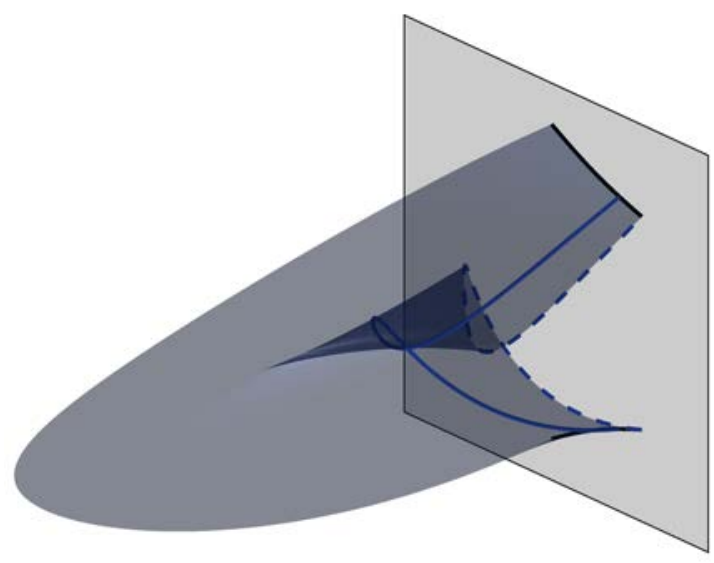

(b) $\omega_{\mathrm{n} 14}<\omega \leq \omega_{\mathrm{n} 12}$ (branch 2 in Fig. 7.3)

Figure 7.2: Invariant manifold for $g<0$ : the solid blue curve is an orbit on the manifold. The dashed line is a nonlinear grazing orbit (of frequency $\omega=\omega_{\mathrm{n} 11}$ for branch 1 or $\omega=\omega_{\mathrm{n} 12}$ for branch 2) forming the boundary of the otherwise unbounded manifold (when $\omega \rightarrow \omega_{\mathrm{nl} 3}$ for branch 1 or $\omega \rightarrow \omega_{\mathrm{nl} 14}$ for branch 2)

As opposed to $g>0$, there are no theoretical statements on the existence of the manifold in the strictly pre-stressed case. The theoretical admissibility criteria obtained for $g>0$ through perturbation near the linear grazing modes, as in [21], are no more available. More importantly, non-existence is proven: the solutions $\mathbf{u}(\cdot ; T, g)$ in the vicinity of the linear grazing orbits are never admissible as proven in Proposition 7.1.

Proposition 7.1 [Non-admissible solutions for strict pre-stress] We assume that there is no internal resonance and all the interaction coefficients are non zero. The periods $T$ of admissible solutions satisfying $0<\left|w_{N}(T)\right|<\infty$ are organized as follow:

1. If $g \neq 0$ and $\mathbf{u}(\cdot ; T,+g)$ is admissible, then $\mathbf{u}(\cdot ; T,-g)$ is not admissible.

2. If $g<0$ and $T>0$ is sufficiently close to $\cup_{j} T_{j} \mathbb{N}^{*}$ then $\mathbf{u}(\cdot ; T, g)$ is not admissible.

3. If $\alpha=\sum_{k=1}^{N} a_{k} \omega_{k}^{-2}>0$ and $T$ is small enough then $\mathbf{u}(\cdot ; T, g)$ is inadmissible.

Above, the first result has a nice interpretation in the FEP. Since the energy does not depend on the sign of $g$, the system features the same FEP for $+g$ and $-g$ as shown by solution (7.2). However, the admissible parts of the curve are distinct for $+g$ and for $-g$. Also, many portions of this curve are neither admissible for $+g$ nor for $-g$, see Figure 7.3. The second result means there is no admissible solution near the harmonics and subharmonics of the linear differential system (2.6a), while the third result says there is no nonlinear modes of high frequency.

Now we turn to prove Proposition 7.1. 


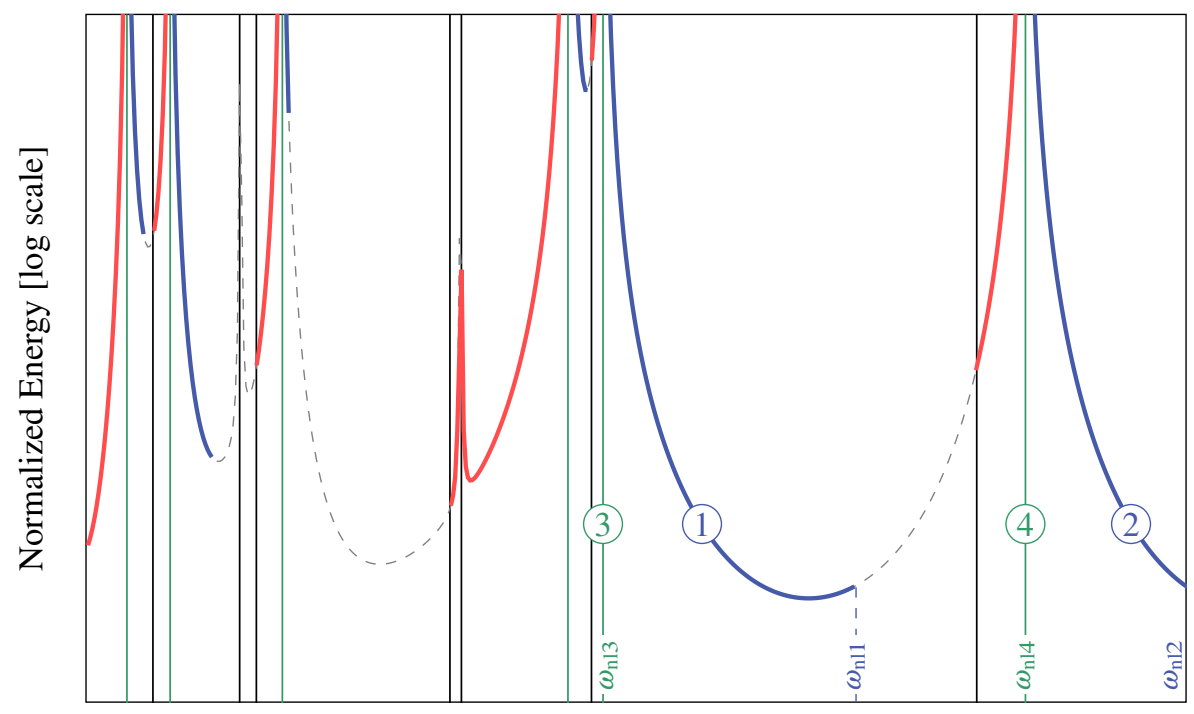

Frequency of vibration $\omega$ [lin scale]

Figure 7.3: FEP — Admissible solutions for $g<0$ [blue spectrum]; admissible solutions for $g>0$ [red spectrum]; non-admissible solutions [thin dashed gray spectrum]. The vertical black lines are the fundamental harmonics and subharmonics of the linear differential system. The vertical green lines show the roots of function $w_{N}(\cdot)$, that is the discrete spectrum for $g=0$. Branches 1 and 2 correspond to the two manifolds depicted in Figures 7.2(a) and 7.2(b). Branches 3 and 4 correspond to the two manifolds displayed in Figures 7.4(a) and 7.4(b). Note that branch 2 becomes inadmissible at high frequencies

Proof. The closed-form solutions to Definition 2.1 are exploited with $g<0$.

1. The proof is valid for $g<0$ and $g>0$. Note that $\mathbf{u}(t ; T,-g)=-\mathbf{u}(t ; T,+g)$. Stating that $\mathbf{u}(t ; T,+g)$ is admissible also means $u_{N}(t ; T,+g) \leq g$ for all time. Accordingly, from (7.3), $u_{N}(t ; T,-g)=$ $-u_{N}(t ; T,+g) \geq-g$ is not admissible. To emphasize, notice that $u_{N}(t ; T, g)<g$ for $t \simeq 0, t \neq 0$ and $u_{N}(t ; T,-g)$ is not admissible since $u_{N}(t ; T,-g)>-g$ for $t \simeq 0, t \neq 0$.

2. The set of initial data for the extended formulation is an analytic set which, for $T=T_{j}$ (a fundamental linear period), recovers the associated linear grazing mode. The mean of a solution is continuously dependent on $T$. A linear solution (without impact) has a 0 mean whereas an admissible solution has a negative mean smaller or equal to $g<0$. Thus, for $g<0$, admissible solutions and their periods and their linear counterparts are necessarily disjointed.

3. The limit for high frequencies is $T=0$ and $\left|w_{N}(0)\right|=\infty$ but, as proven in Section 5.3 with the two key assumptions "no internal resonance" and "non-zero interaction coefficients", the manifold of initial data $\mathcal{V}_{0}=\left\{\left(\mathbf{u}(0 ; T, g), \dot{\mathbf{u}}^{+}(0 ; T, g)\right), 0<\left|w_{N}(T)\right|<\infty\right\}$ is analytic with analytic extension when $\left|w_{N}(T)\right|=\infty$. It is known that the solution to (2.5) is continuously dependent on the initial data [3]. To prove the inadmissibility, it then suffices to show that $\mathbf{u}(t ; 0, g)>g$ for some time $t$ since by continuity the same remains true for $\mathbf{u}(t ; T, g)>g$ and $T$ small enough. Using the proof of Proposition 5.2, it is known that $\dot{u}_{N}^{+}(0 ; T, g) \sim(T g) /(2 \alpha)<0$ and $\ddot{u}_{N}^{+}(0 ; T, g) \sim-g / \alpha>0$ thus, for $T=0, \dot{u}_{N}^{+}(0 ; 0, g)=0$ and $\ddot{u}_{N}^{+}(0 ; 0, g)>0$, which is an evidence of inadmissibility since $u_{N}(t ; 0, g)>g$ for $t \gtrsim 0$.

7.2. Static grazing The grazing case $g=0$ is unique and quite different from $g \neq 0$. The nonlinear spectrum becomes discrete, in a way similar to the purely linear system (2.6a). The periods of the extended formulation are then roots of the function $w_{N}(\cdot)$ given in Eq. (3.1) (green vertical asymptotes in Figure 7.3).

Proposition 7.2 [Static grazing: solutions to the extended formulation] When $g=0$, the period of the solutions to the extended formulation are the roots of $w_{N}$ and the spectrum is countable. Moreover, to every root of $w_{N}$ is associated a one-parameter continuous family of periodic orbits of the same period:

$$
\begin{aligned}
& \mathbf{u}(0 ; T)=v \mathbf{w}(T) \quad \text { with } \quad u_{N}(0 ; T)=w_{N}(T)=0, \\
& \dot{\mathbf{u}}(0 ; T)=v \mathbf{e}_{N} .
\end{aligned}
$$

The admissibility condition implies $v \geq 0$.

Proof. From Lemma 4.1 the solutions to the extended formulation read $\mathbf{u}(0 ; T)=v \mathbf{w}(T)$ and $\dot{\mathbf{u}}(0 ; T)=v \mathbf{e}_{N}$, from which $u_{N}(0 ; T)=g=0=v w_{N}(T)$ is deduced. The case $v \neq 0$ is assumed; otherwise the static 

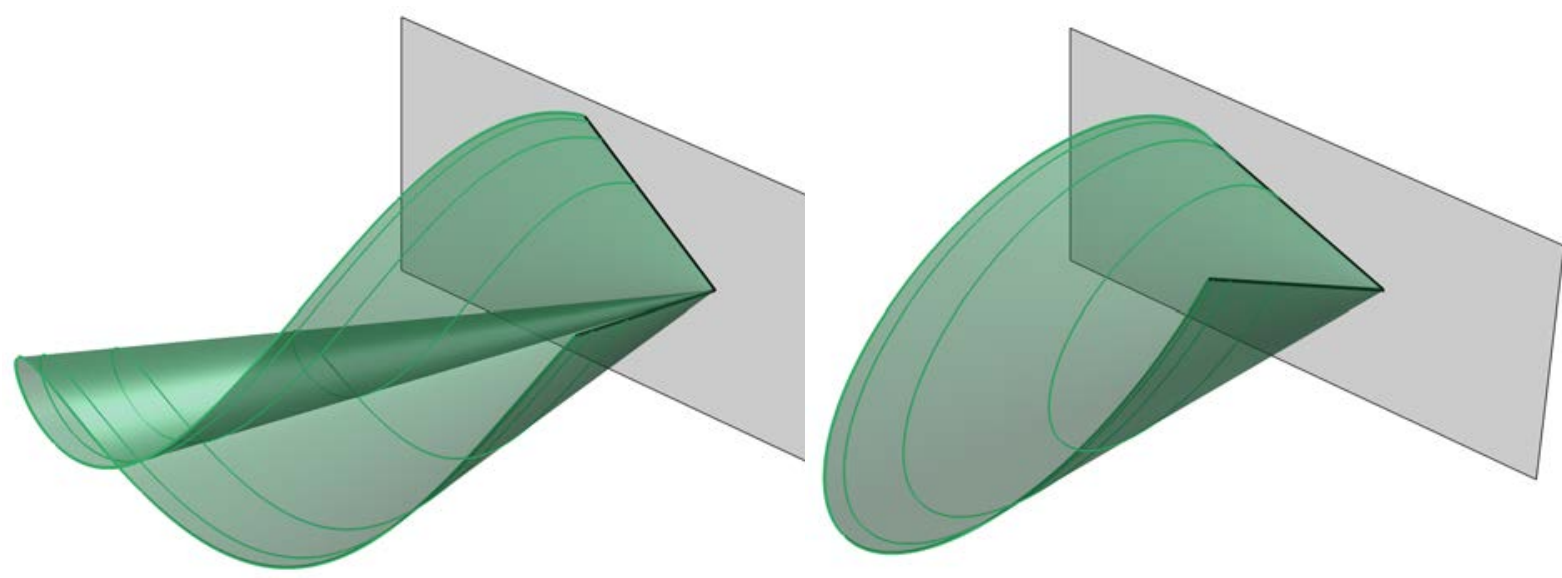

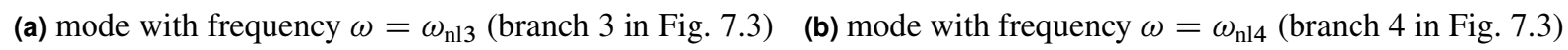

Figure 7.4: Invariant manifold for $g=0$. Solid lines show orbits on the manifold. They are all of the same frequency for a given manifold

equilibrium solution is retrieved which is not of interest. Accordingly, $g=0$ implies $w_{N}(T)=0$. Moreover, $\dot{u}_{N}^{+}(0 ; T)=v$ and the admissibility condition is satisfied with a positive incoming velocity $v>0$. For $v=0$, the solution is genuinely admissible since it is the equilibrium. So the solution satisfies $u_{N}(t ; T) \leq g$ for $v \geq 0$ and $t \approx 0$ (or $T$ by symmetry).

It is important to realize that this (nonlinear) spectrum is not the countably finite spectrum $\left\{\omega_{1}, \ldots, \omega_{N}\right\}$ of the linear system. Instead, it is related to the roots of $w_{N}(T)$. In general, $w_{N}(T)$ is a non-periodic analytic function of $T$ and shows, at most, a (possibly infinite) countable set of roots, as illustrated in Figure 3.1.

Lemma 7.1 [Infinite spectrum] If $a_{k}>0$ for all $k \in\{1, \ldots, N\}$ then the set of roots of $w_{N}$ is a countably infinite set.

Proof. For $T>0, w_{N}$ is singular $\left(\left|w_{N}(T)\right|=\infty\right)$ only when $T=\ell T_{k}$ for some $\ell \in \mathbb{N}$ and $k \in\{1, \ldots, N\}$. Previous computations show that for $T \approx \ell T_{k}, T \neq \ell T_{k}$ :

$$
w_{N}(T) \sim a_{k} \phi_{k}(T) \sim \frac{2 a_{k}}{\omega_{k}^{2}} \frac{1}{T-\ell T_{k}} .
$$

Therefore, $w_{N}$ changes its sign through a singularity. Still, it is continuous on $\left.\mathcal{D}=\right] 0 ;+\infty\left[-\cup_{j=1}^{N} T_{j} \mathbb{N}\right.$, and on every connex component $I=] i T_{j} ; \ell T_{k}$ [ with vertical asymptotes on its boundary $\partial I=\left\{i T_{j}, \ell T_{k}\right\}$. Since $a_{j}$ and $a_{k}$ have the same positive sign, $w_{N}^{+}\left(i T_{j}\right)=+\infty$ and $w_{N}^{-}\left(\ell T_{k}\right)=-\infty, w_{N}$ has at least one root in $I$. The set of connex components of $\mathcal{D}$ is infinite, so the set of roots of $w_{N}$ too.

It can be demonstrated numerically that many of the calculated roots yield non-physical solutions: only a few exhibit a last mass on the left side of the wall for all times.

8. Multiple oscillators and self-contact The above presentation can be extended to other configurations that might be of mechanical interest:

- Unilateral contact with the wall is enforced on a degree-of-freedom other than the last one. This is succinctly discussed in [27] for a two-dof system where periodic solutions with one sticking phase per period on mass 2 are related to periodic solutions with one impact per period on mass 1.

- Initially separated $N_{1}$ - and $N_{2}$-dof chains interacting through impact-driven conditions. Let us denote by indices $k_{1}$ and $k_{2}$ the two interacting dofs, from chain 1 and chain 2 respectively as depicted in Figure 8.1(a) (with $k_{1}=2$ and $k_{2}=3$ ), then

$$
u_{k_{1}}(t)-u_{k_{2}}(t) \leq g,
$$

where the positive gap $g$ represents the distance between the equilibrium position of the two masses subjected to possible contact occurrences. The impact law is classically expressed through the conservation of linear momentum and conservation of kinetic energy at the impact time such that 


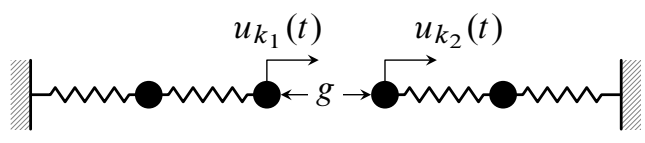

(a) Multiple oscillators

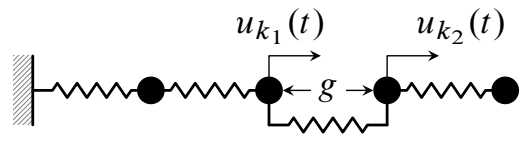

(b) Self-contact

Figure 8.1: Other potential (simplified) configurations of interest

$u_{k_{1}}(t)-u_{k_{2}}(t)=g$ implies $\dot{u}_{k_{1}}^{+}(t)=2 V(t)-\dot{u}_{k_{1}}^{-}(t)$ and $\dot{u}_{k_{2}}^{+}(t)=2 V(t)-\dot{u}_{k_{2}}^{-}(t)$ where $V(t)$ is the velocity of the center of mass, which yields

$$
\left(m_{k_{1}}+m_{k_{2}}\right) V(t)=m_{k_{1}} \dot{u}_{k_{1}}^{ \pm}(t)+m_{k_{2}} \dot{u}_{k_{2}}^{ \pm}(t) .
$$

Using the orthogonal change of variables $u_{k_{1}}^{*}=u_{k_{1}}-u_{k_{2}}$ and $u_{k_{2}}^{*}=u_{k_{1}}+u_{k_{2}}$, new mass and stiffness matrices $\mathbf{M}^{*}$ and $\mathbf{K}^{*}$ with the same (natural) frequencies (as $\mathbf{M}$ and $\mathbf{K}$ ) shall be built. The problem to be solved thus reduces to the formulation explained previously with the unilateral condition

$$
u_{k_{1}}^{*} \leq g
$$

However, the new system after this change of basis does not correspond to a chain since the mass matrix $\mathbf{M}^{*}$ is not diagonal.

- Self-contact in the same chain of masses, where two neighboring dof should satisfy a contact constraint, see Figure 8.1(b).

9. Conclusion Nonlinear normal modes of conservative multi-degrees-of-freedom systems are one-parameter continuous families of periodic motions. They have been investigated since the 1950's for vibratory systems involving smooth and differentiable nonlinearities. In this work, an extension to a vibro-impact system exhibiting non-differentiable displacement and discontinuous velocity is proposed. Based on the construction of a convenient Poincare map and with the assumption of a single impact per period, (quasi-) closed-form solutions are obtained and nonlinear modes of vibration are shown to exist. When the initial gap separating the oscillator from the impacted rigid foundation is positive, they can be visualized as piecewise analytic two-dimensional manifolds in the state-space, formed by the union of one "flat" submanifold (contact is not activated and the normal modes are invariant elliptic disks) and one "curved" submanifold (contact occurs once per period) sharing a common grazing orbit. From the expressions for the normal modes, the frequency-energy relationships on each mode are captured and are (obviously) of piecewise type. Interestingly, it is shown that the manifolds emanating from the linear modes of period $T_{i}$ and from the subharmonics of the linear modes of period $n T_{i}, n \in \mathbb{N}^{+}$ and $i \in\{1, \ldots, N\}$ are distinct. Altogether, such manifolds "shape" the state-space of the system and graphically illustrate the dynamical complexities induced by the presence of a perfectly elastic impact law into the (otherwise linear) governing equations.

A succinct spectral stability analysis is conducted for the first nonlinear mode. It is numerically demonstrated that an intricate pattern of stable and unstable solutions arises as the frequency of the periodic solution is increased. The three commonly reported bifurcation mechanisms are found: they depend on where the eigenvalues of the linearized First Return Map leave the unit circle in the complex plane. Other more advanced bifurcations involving grazing orbits transitioning from a single impact per period to multiple impacts per period are conjectured.

The linear "flat" portions of the manifolds for pre-stressed or statically grazing systems cease to exist. More importantly, the corresponding "essentially" nonlinear manifolds cannot be found as continuations of linear ones.

Finally, it is briefly explained that systems embedding multiple oscillators or involving self-contact can also be tackled through a simple change of variables.

Appendix A. Stability analysis through perturbed orbits The stability analysis of a periodic orbit lying on the previously constructed manifolds as explained in Corollary 3.1 is revisited in a more pragmatic and intuitive manner. Let's first define the notation $\mathbf{U}_{t}:=\left(\mathbf{u}_{t}, \dot{\mathbf{u}}_{t}\right):=\left(\mathbf{u}(t), \dot{\mathbf{u}}^{+}(t)\right)$. The initial condition on the Poincaré section of this periodic orbit is denoted by $\mathbf{U}_{0}$ and its period, by $T_{0}$. By definition of the Poincaré section, $\overline{\mathbf{e}}_{N}^{\top} \mathbf{U}_{0}=u_{N}(0)=g$. We want to know how slightly disturbed initial conditions 
$\mathbf{U}_{0}+\delta \mathbf{U}_{0}$ defined on the Poincaré section, i.e. satisfying $\overline{\mathbf{e}}_{N}^{\top} \delta \mathbf{U}_{0}=0$, are mapped back onto the Poincaré section $\mathbf{U}_{T}=\mathbf{U}_{0}+\delta \mathbf{U}_{T}$ after a time $T=T_{0}+\delta T$ with the condition $\overline{\mathbf{e}}_{N}^{\top} \delta \mathbf{U}_{T}=0$. If their amplitude is magnified, then the periodic orbit is unstable. The Poincaré map (2.27) in physical coordinates reads

$$
\left(\begin{array}{c}
\mathbf{u}(T) \\
\dot{\mathbf{u}}^{+}(T)
\end{array}\right)=\left[\begin{array}{cc}
\mathbf{P} \cos (T \boldsymbol{\Omega}) \mathbf{P}^{-1} & \mathbf{P} \boldsymbol{\Omega}^{-1} \sin (T \boldsymbol{\Omega}) \mathbf{P}^{-1} \\
-\mathbf{S P} \boldsymbol{\Omega} \sin (T \boldsymbol{\Omega}) \mathbf{P}^{-1} & \mathbf{S P} \cos (T \boldsymbol{\Omega}) \mathbf{P}^{-1}
\end{array}\right]\left(\begin{array}{c}
\mathbf{u}(0) \\
\dot{\mathbf{u}}^{+}(0)
\end{array}\right)=\left[\begin{array}{c}
\boldsymbol{\Gamma}_{1}(T) \boldsymbol{\Gamma}_{2}(T) \\
\boldsymbol{\Gamma}_{3}(T) \boldsymbol{\Gamma}_{4}(T)
\end{array}\right]\left(\begin{array}{c}
\mathbf{u}(0) \\
\dot{\mathbf{u}}^{+}(0)
\end{array}\right)
$$

that is $\boldsymbol{\Gamma}(T)=\overline{\mathbf{P}} \mathbf{R}(T) \overline{\mathbf{P}}^{-1}$. An admissible perturbed orbit starting on the Poincaré section at $t=0$ in a neighborhood of the actual periodic orbit and coming back to the Poincaré section at $T=T_{0}+\delta T$ is thus governed by the system

$$
\left(\begin{array}{c}
\mathbf{u}_{0}+\delta \mathbf{u}_{T} \\
\dot{\mathbf{u}}_{0}+\delta \dot{\mathbf{u}}_{T}
\end{array}\right)=\left[\begin{array}{ll}
\boldsymbol{\Gamma}_{1}\left(T_{0}+\delta T\right) & \boldsymbol{\Gamma}_{2}\left(T_{0}+\delta T\right) \\
\boldsymbol{\Gamma}_{3}\left(T_{0}+\delta T\right) & \boldsymbol{\Gamma}_{4}\left(T_{0}+\delta T\right)
\end{array}\right]\left(\begin{array}{c}
\mathbf{u}_{0}+\delta \mathbf{u}_{0} \\
\dot{\mathbf{u}}_{0}+\delta \dot{\mathbf{u}}_{0}
\end{array}\right)
$$

with the property

$$
\mathbf{e}_{N}^{\top} \delta \mathbf{u}_{T}=\mathbf{e}_{N}^{\top} \delta \mathbf{u}_{0}=0 .
$$

Neglecting higher order terms, system (A.2) simplifies to

$$
\left(\begin{array}{l}
\mathbf{u}_{0}+\delta \mathbf{u}_{T} \\
\dot{\mathbf{u}}_{0}+\delta \dot{\mathbf{u}}_{T}
\end{array}\right)=\left[\begin{array}{ll}
\boldsymbol{\Gamma}_{1}\left(T_{0}\right)+\dot{\boldsymbol{\Gamma}}_{1}\left(T_{0}\right) \delta T & \boldsymbol{\Gamma}_{2}\left(T_{0}\right)+\dot{\boldsymbol{\Gamma}}_{2}\left(T_{0}\right) \delta T \\
\boldsymbol{\Gamma}_{3}\left(T_{0}\right)+\dot{\boldsymbol{\Gamma}}_{3}\left(T_{0}\right) \delta T & \boldsymbol{\Gamma}_{4}\left(T_{0}\right)+\dot{\boldsymbol{\Gamma}}_{4}\left(T_{0}\right) \delta T
\end{array}\right]\left(\begin{array}{c}
\mathbf{u}_{0}+\delta \mathbf{u}_{0} \\
\dot{\mathbf{u}}_{0}+\delta \dot{\mathbf{u}}_{0}
\end{array}\right)
$$

Since Eq. (A.1) is satisfied by the periodic orbit, Eq. (A.4) becomes

$$
\left(\begin{array}{l}
\delta \mathbf{u}_{T} \\
\delta \dot{\mathbf{u}}_{T}
\end{array}\right)=\left[\begin{array}{ll}
\boldsymbol{\Gamma}_{1}\left(T_{0}\right) & \boldsymbol{\Gamma}_{2}\left(T_{0}\right) \\
\boldsymbol{\Gamma}_{3}\left(T_{0}\right) & \boldsymbol{\Gamma}_{4}\left(T_{0}\right)
\end{array}\right]\left(\begin{array}{l}
\delta \mathbf{u}_{0} \\
\delta \dot{\mathbf{u}}_{0}
\end{array}\right)+\delta T\left[\begin{array}{ll}
\dot{\boldsymbol{\Gamma}}_{1}\left(T_{0}\right) & \dot{\boldsymbol{\Gamma}}_{2}\left(T_{0}\right) \\
\dot{\boldsymbol{\Gamma}}_{3}\left(T_{0}\right) & \dot{\boldsymbol{\Gamma}}_{4}\left(T_{0}\right)
\end{array}\right]\left(\begin{array}{l}
\mathbf{u}_{0} \\
\dot{\mathbf{u}}_{0}
\end{array}\right) .
$$

From Eq. (A.3), we can express $\delta T$ in terms of $\left(\delta \mathbf{u}_{0}, \delta \dot{\mathbf{u}}_{0}\right)$ since

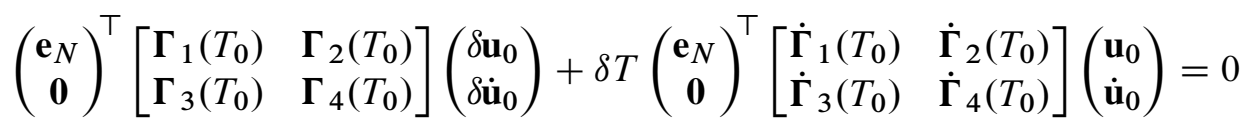

which leads to

$$
\delta T=-\frac{\overline{\mathbf{e}}_{N}^{\top} \boldsymbol{\Gamma}\left(T_{0}\right) \delta \mathbf{U}_{0}}{\mathbf{\mathbf { e }}_{N}^{\top} \dot{\Gamma}\left(T_{0}\right) \mathbf{U}_{0}} .
$$

System (A.5) thus reads

$$
\delta \mathbf{U}_{T}=\left(\boldsymbol{\Gamma}-\frac{\dot{\boldsymbol{\Gamma}}\left(T_{0}\right) \mathbf{U}_{0} \overline{\mathbf{e}}_{N}^{\top} \boldsymbol{\Gamma}\left(T_{0}\right)}{\overline{\mathbf{e}}_{N}^{\top} \dot{\boldsymbol{\Gamma}}\left(T_{0}\right) \mathbf{U}_{0}}\right) \delta \mathbf{U}_{0}
$$

or, in a compact form

$$
\delta \mathbf{U}_{T}=\delta \Gamma \delta \mathbf{U}_{0}
$$

where $\delta \boldsymbol{\Gamma}$ is a notation. The matrices $\delta \boldsymbol{\Gamma}$ in Eq. (A.9) and $\mathbf{D F}$ in Eq. (3.5) are two expressions of the same linearized First Return Map, in physical and modal coordinates, respectively (see transformation (4.3) between the two systems). They both exhibit the additional extraneous eigenvalue 0 which should be discarded. To this end, let's consider the rectangular mapping $\mathbf{Q}_{N}^{\top}$ which removes row $N$ of a vector it multiplies; $\mathbf{Q}_{N}$ is a rectangular matrix of size $2 N \times 2 N-1$ storing "1" on its "fake diagonal". Let's also use two new reduced quantities: $\mathbf{U}_{0, N}=\mathbf{Q}_{N} \mathbf{U}_{0}$ is $\mathbf{U}_{0}$ with its $N$ th coordinate removed; same for $\mathbf{U}_{T, N}$ with $\mathbf{U}_{T}$, as well as their corresponding perturbations. System (A.9) simplifies to

$$
\delta \mathbf{U}_{T, N}=\mathbf{Q}_{N}^{\top} \delta \boldsymbol{\Gamma} \mathbf{Q}_{N} \delta \mathbf{U}_{0, N}=\delta \boldsymbol{\Gamma}_{N} \delta \mathbf{U}_{0, N} .
$$

A similar reduction could be proposed for $\mathbf{D F}$ in the modal coordinates system. The eigenvalues of the reduced maps dictate the instability of the targeted periodic orbit. For a given periodic response, a 
sufficient condition for instability is that at least one of these eigenvalues in modulus lies outside the unit circle in the complex plane.

Appendix B. Eigenvalues of the Return Map Matrix The spectrum of the Return Map Matrix $\mathbf{R}(T)$ lies on the unit circle. Unfortunately, this is not sufficient to conclude on the stability of the nonlinear modes but it could be useful for further works on this topic. It is determined by finding $\lambda$ such that

$$
\operatorname{det}\left(\left[\begin{array}{cc}
\cos (T \Omega)-\lambda \mathbf{I} & \mathbf{\Omega}^{-1} \sin (T \boldsymbol{\Omega}) \\
-\mathcal{S} \boldsymbol{\Omega} \sin (T \Omega) & \mathcal{S} \cos (T \Omega)-\lambda \mathbf{I}
\end{array}\right]\right)=0
$$

The first row of blocks stores diagonal matrices and the determinant can be calculated blockwise

$$
\operatorname{det}\left((\cos (T \Omega)-\lambda \mathbf{I})(\mathcal{S} \cos (T \Omega)-\lambda \mathbf{I})+\mathcal{S} \sin (T \Omega)^{2}\right)=0
$$

or equivalently

$$
\operatorname{det}\left(\mathcal{S}-\lambda \cos (T \boldsymbol{\Omega})(\mathcal{S}+\mathbf{I})+\lambda^{2} \mathbf{I}\right)=0 .
$$

We can respectively premultiply and postmultiply by the non-singular matrices $\mathbf{P}$ and $\mathbf{P}^{-1}$ such that

$$
\operatorname{det} \mathbf{P} \operatorname{det}\left(\mathcal{S}-\lambda \cos (T \boldsymbol{\Omega})(\mathcal{S}+\mathbf{I})+\lambda^{2} \mathbf{I}\right) \operatorname{det} \mathbf{P}^{-1}=0
$$

which is equivalent to $\operatorname{det}\left(\mathbf{P}\left(\mathcal{S}-\lambda \cos (T \Omega)(\mathcal{S}+\mathbf{I})+\lambda^{2} \mathbf{I}\right) \mathbf{P}^{-1}\right)=0$ which itself simplifies to

$$
\operatorname{det}\left(\mathbf{S}-\lambda \cos (T \mathbf{\Omega})(\mathbf{S}+\mathbf{I})+\lambda^{2} \mathbf{I}\right)=0 .
$$

This becomes the determinant of a diagonal system with the following discussion:

1. entries $i=1, \ldots, N-1: 1-2 \lambda \cos \left(\omega_{i} T\right)+\lambda^{2}=0$;

2. entry $N:-1+\lambda^{2}=0$.

All eigenvalues are of modulus 1 .

\section{References}

[1] Vincent Acary and Bernard Brogliato, Numerical Methods for Non-smooth Dynamical Systems, vol. 35 of Lecture Notes in Applied and Computational Mechanics, Springer, 2008, ISBN: 9783540753919.

[2] HÉDY ATTOUCH, Variational convergence for functions and operators, Pitman, 1984, ISBN: 9780273085836.

[3] PATRICK BAllard, The dynamics of discrete mechanical systems with perfect unilateral constraints, Archive for Rational Mechanics and Analysis, 154 (2000), pp. 199-274, [hal-00111308].

[4] Jérôme Bastien, Frédéric Bernardin, and Claude-Henri Larmarque, Non Smooth Deterministic or Stochastic Discrete Dynamical Systems, Mechanical Engineering and Solid Mechanics Series, Wiley, 2013, ISBN: 9781848215252.

[5] Olivier BrÜls, Vincent ACARy, AND Alberto CARDona, Simultaneous enforcement of constraints at position and velocity levels in the nonsmooth generalized $\alpha$-scheme, Computer Methods in Applied Mechanics and Engineering, 281 (2014), pp. 131-161, [hal-01059823].

[6] ShyH-Leh Chen And Steven Shaw, Normal modes for piecewise linear vibratory systems, Nonlinear Dynamics, 10 (1996), pp. 135-164, [hal-01354038].

[7] Mario di Bernardo, Alan Champneys, Christopher Budd, and Piotr Kowalczyk, Piecewise-smooth Dynamical Systems: Theory and Applications, vol. 163 of Applied Mathematical Sciences, Springer, 2008, ISBN: 9781846280399.

[8] David Doyen, Alexandre Ern, And Serge Piperno, Time-integration schemes for the finite element dynamic Signorini problem, SIAM Journal of Scientific Computing, 33 (2011), pp. 223-249, [hal-00440128].

[9] RaOuf Ibrahim, Vibro-impact Dynamics: Modeling, Mapping and Applications, vol. 43 of Lecture Notes in Applied and Computational Mechanics, Springer, 2009, ISBN: 9783642002748.

[10] AleXANDER IVANOv, The stability of periodic solutions of discontinuous systems that intersect several surfaces of discontinuity, Journal of Applied Mathematics and Mechanics, 62 (1998), pp. 677-685, [hal-01354035].

[11] Dongying Jiang, Christophe Pierre, And Steven Shaw, Large-amplitude non-linear normal modes of piecewise linear systems, Journal of Sound and Vibration, 272 (2004), pp. 869-891, [hal-01350797].

[12] StÉPHANE JUNCA AND BERNARD ROUSSELET, The method of strained coordinates for vibrations with weak unilateral springs, IMA Journal of Applied Mathematics, 76 (2010), pp. 251-276, [hal-00395351].

[13] STÉPHANE JUNCA AND LY TONG, Limitation on the method of strained coordinates for vibrations with weak grazing unilateral contact, Nonlinear Dynamics, 80 (2015), pp. 197-207, [hal-01143612]. 
[14] Gaëtan Kerschen, Maxime Peeters, Jean-Claude Golinval, and Alexander Vakakis, Nonlinear normal modes, part I: A useful framework for the structural dynamicist, Mechanical Systems and Signal Processing, 23 (2009), pp. 170-194, [hal-01357931].

[15] Denis Laxalde And Mathias Legrand, Nonlinear modal analysis of mechanical systems with frictionless contact interfaces, Computational Mechanics, 47 (2011), pp. 469-478, [hal-00492775].

[16] Mathias Legrand, Dongying Jiang, Christophe Pierre, and Steven Shaw, Nonlinear normal modes of a rotating shaft based on the invariant manifold method, International Journal of Rotating Machinery, (2002), [hal-01315570].

[17] LeOnARD MeIrovitch, Elements of Vibration Analysis, McGraw-Hill, 1975, ISBN: 9780070413405.

[18] Kenneth Meyer, Glen Hall, And Dan Offin, Introduction to Hamiltonian Dynamical Systems and the N-Body Problem, Springer, 2009, ISBN: 9780387097237.

[19] El Hadi Moussi, Sergio Bellizzi, Bruno Cochelin, ANd Ionel Nistor, Nonlinear normal modes of a two degree-of-freedom piecewise linear system, Mechanical Systems and Signal Processing, 64-65 (2015), pp. 266-281, [hal-00783088].

[20] Ali Hasan NAYFeh AND Dean Mook, Nonlinear Oscillations, Wiley-VCH Verlag GmbH, 2007, ISBN: 9783527617586.

[21] ARNE NORDMARK, Existence of periodic orbits in grazing bifurcations of impacting mechanical oscillators, Nonlinearity, 14 (2001), pp. 1517-1542, [hal-01297283].

[22] Madeleine PASCAL, Dynamics and stability of a two-degree-of-freedom oscillator with an elastic stop, Journal of Computational and Nonlinear Dynamics, 1 (2005), pp. 94-102, [hal-00342874].

[23] VAlery PilipCHUK, Impact modes in discrete vibrating systems with rigid barriers, International Journal of Non-Linear Mechanics, 36 (2001), pp. 999-1012, [hal-01295245].

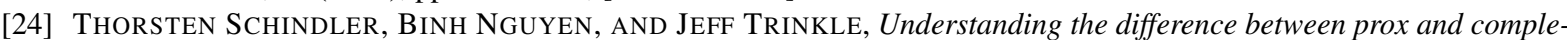
mentarity formulations for simulation of systems with contact, in IEEE/RSJ International Conference on Intelligent Robots and Systems, San Fransisco, 2011, pp. 1433-1438, [hal-01309169].

[25] RÜDIGER SEYDEL, Practical Bifurcation and Stability Analysis, vol. 5 of Interdisciplinary Applied Mathematics, Springer, 2010, ISBN: 9781441917393.

[26] StEven Shaw, An invariant manifold approach to nonlinear normal modes of oscillation, Journal of Nonlinear Science, 4 (1994), pp. 419-448, [hal-01354040].

[27] Huong Le Thi, Stéphane Junca, and Mathias Legrand, Periodic solutions of a two-degree-of-freedom autonomous vibro-impact oscillator with sticking phases, preprint, (2016), [hal-01305719].

[28] ANders Thorin, MATHIAs Legrand, AND STÉPHANE JUnCA, Nonsmooth modal analysis: investigation of a 2-dof spring-mass system subject to an elastic impact law, in Proceedings of the ASME International Design Engineering Technical Conferences and Computers and Information in Engineering Conference, Boston, 2015, [hal-01185973]. DETC2015-46796.

[29] Cyril Touzé, Olivier Thomas, AND Antoine Chaigne, Hardening/softening behaviour in non-linear oscillations of structural systems using non-linear normal modes, Journal of Sound and Vibration, 273 (2004), pp. 77-101, [hal-00830693].

[30] AlEXANDER VAKAKIS, Non-linear normal modes and their applications in vibration theory: an overview, Mechanical Systems and Signal Processing, 11 (1997), pp. 3-22, [hal-01354037].

[31] Alexander Vakakis, Leonid Manevitch, Yuri Mikhlin, Valery Pilipchuk, and Alexandr Zevin, Normal Modes and Localization in Nonlinear Systems, John Wiley \& Sons, 1996, ISBN: 9780471133193. 